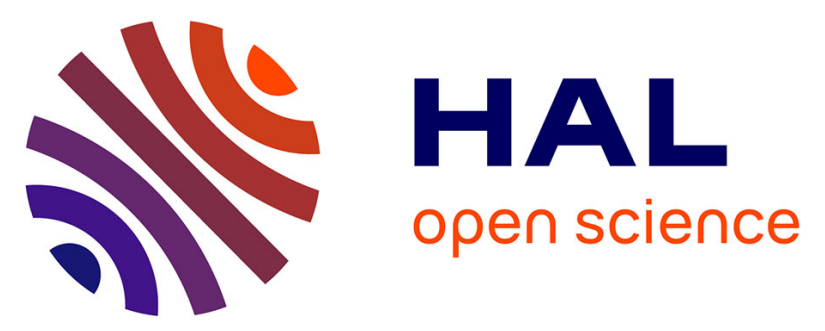

\title{
Improving manufacturing of aeronautical parts with an enhanced industrial Robotised Fibre Placement Cell using an external force-vision scheme
}

\author{
Maylis Uhart, Olivier Patrouix, Yannick Aoustin
}

\section{- To cite this version:}

Maylis Uhart, Olivier Patrouix, Yannick Aoustin. Improving manufacturing of aeronautical parts with an enhanced industrial Robotised Fibre Placement Cell using an external force-vision scheme. International Journal on Interactive Design and Manufacturing, 2015, p., 1-21, Print ISSN 1955-2513, Online ISSN 1955-2505. 10.1007/s12008-015-0271-6 . hal-01179004

\author{
HAL Id: hal-01179004 \\ https://hal.science/hal-01179004
}

Submitted on 21 Jul 2015

HAL is a multi-disciplinary open access archive for the deposit and dissemination of scientific research documents, whether they are published or not. The documents may come from teaching and research institutions in France or abroad, or from public or private research centers.
L'archive ouverte pluridisciplinaire HAL, est destinée au dépôt et à la diffusion de documents scientifiques de niveau recherche, publiés ou non, émanant des établissements d'enseignement et de recherche français ou étrangers, des laboratoires publics ou privés. 


\title{
Improving manufacturing of aeronautical parts with an enhanced industrial Robotised Fibre Placement Cell using an external force-vision scheme
}

\author{
M. Uhart • O. Patrouix • Y. Aoustin
}

Received: date / Accepted: date

\begin{abstract}
Composite materials are increasingly used in the demanding field of aeronautics. To meet this growing need, the company Coriolis Composites has developed an Automated Fibre Placement device already on the market. This process uses an industrial manipulator robot. This fibre placement task requires a compacting strength adapted to the material used. For the robot, the reference trajectories in position of each tape are prescribed off-line. However, accuracy problems of tape placement appear when off-line programmed robot trajectories. The proposed approach to address the accuracy problem and the manufacturing constraints is an interactive manufacturing scheme based on exteroceptive sensor loops. This paper shows an interactive manufacturing approach based on force servoing to control the compacting strength and on visual servoing to control the lateral position of the tape in order to improve the fibre placement accuracy and then the quality of manufactured parts.
\end{abstract}

Keywords Composite manufacturing - Robot . Quality improvement · Force-Vision control · Sensor integration · Interactive Manufacturing

M. Uhart

ESTIA, ESTIA Recherche, Technopole Izarbel, 64210 Bidart, France

L'UNAM, IRCCyN de Nantes UMR CNRS 6597, 1 rue de la Noë, 44321 Nantes Cedex 3, France

E-mail: m.uhart@estia.fr, Maylis.Uhart@irccyn.ec-nantes.fr

O. Patrouix

ESTIA, ESTIA Recherche, Technopole Izarbel, 64210 Bidart, France

E-mail: o.patrouix@estia.fr

Y. Aoustin

L'UNAM, IRCCyN de Nantes UMR CNRS 6597, 1 rue de la Noë, 44321 Nantes Cedex 3, France

E-mail: Yannick.Aoustin@irccyn.ec-nantes.fr

\section{Introduction}

Composite materials have many advantages. Their behavior is very beneficial for industry $[12,6]$. The lifespan of this kind of materials is enhanced because of the good resistance to fatigue. They have good mechanical characteristics such as resistance to corrosion and enhancement of resistance to fire. Then, this material is lighter that classical materials. So, it can reduce the weight of products and the fuel consumption. It is easy to obtain complex shapes because of the design flexibility of the composite. The manufacturing cost is higher than for traditional materials such as steel, wood and aluminium but the lifespan is better. Consequently, there is a good depreciation.

Thus, more and more industries use, or think to use, this material to manufacture their mechanical structures in various fields like planes, automotive, marine, sport and wind energy. This is a new fast growing market [37]. In 2012, Europe has consumed most of composites in the following areas: Aerospace and defense (46\% of $8100 \mathrm{t}$ ), Wind Turbines $(74 \%$ of $9500 \mathrm{t}$ ) and Automotive (56\% of $2150 \mathrm{t}$ ). Asia has consumed most of composites in sport and leisure (86\% of $7800 \mathrm{t}$ ).

There are several methods for manufacturing composite parts [20]. The hand lay-up is the simplest and oldest method of the manufacturing process using composite. The carbon ply is positioned manually in the mould. Despite the high level of skills of the composite worker, there is a lack of repeatability on large and complex structure. On the contrary, the automated tape placement has got a better repeatability. A single and wide tape is deposited on the mould with the help of a computer numerical control (CNC) machine tool. Thus, the production efficiency is improved and the cost is reduced. Research work has been carried on the auto- 
mated fibre placement process to use manipulator arm in order to have a better accessibility and a good repeatability [22]. Thus, the robot can lay-up on workpiece with double curvature.

More and more industrial tasks are robotised such as grinding, deburring or polishing [29,24]. First, the manual task must be analysed before being automatised. A task is defined in degrees of freedom needed to achieve it. The compatibility between the robot and the task must be checked. The number of degrees of freedom of the tool must be greater or equal to the number of degrees of freedom of the task. Consequently, research work should be done in order to improve the new process and its accuracy taking into account all the parameters of the fibres placement task.

It is possible to equip the system with sensors in order to add information on the interaction with the environment and/or the location in the environment. To do that, a sensor-based control must be designed. The sensors can be a force sensor [40] or a visual system [10] according to the process.

The force sensor is generally attached on the wrist of the robot between the tool and the manipulator arm. The force feedback is already used and tested in the frame of surgical applications [39]. In this way, tool-path can be corrected in accordance with the force measured. This kind of control is implemented in some applications such as grinding or deburring [29]. Controlling a robot in contact has evolved considerably in recent years [36]. It can be designed according to different architectures [9] that can be classified into two approaches. The first approach is the command without force setpoint where the robot is only controlled in position $[30,14]$. The second approach is the command with force setpoint where the robot is controlled in force or position according to the direction. It can be an hybrid force-position command [28] or an external hybrid command [17].

Concerning the visual system, it can be composed of one or more cameras and it can be fixed [18] or embedded [31] on the manipulator arm according to the point of view desired. Because we work with carbon fibres whose the colour is black, it is interesting to use a high speed camera with a laser [26]. The structured light allows capturing the 3D shape information on the part by analysing the intersection between the light pattern and the part [38]. The visual-based command consists in using visual information from a visual system to control a robotic system $[7,16]$. This kind of command allows the trajectory tracking, the seizure or the object insertion according the application. The positioning errors are offset through the closed control loop on data extracted from the image. It can be a direct visual ser- voing or an indirect visual servoing [25].

Finally, force and vision can be combined to have more informations on the system during the process [15]. Two impedance control structures based on visual servo control and on force control are proposed in [5]. The force control are carried out from the measured physical force or from a fictitious force obtained through the visual information. They allows the tasks range of the robot to be extended to partially structured environments.

In our case, we have to study the task of laying-up and the process developed by the company Coriolis Composites. It has developed a robot based solution for the AFP between 1996 and 2001. It markets since 2001. About twenty machines were sold for aerospace applications. It has partnered with other companies that use this new system and universities in order to improve the process within the framework of research and knowledge transfer programs named IMPALA for Innovation Materials and Process of fibres plAcement with LAser. However, we have some constraints. The company used a maximum of already existing components for the solution. In aeronautics, the number of parts made of composite is increasing and the accuracy constraints are important. For example, it is forbidden to have recovered fibres, the space between two fibres and the angular deviation must respect the specifications defined by the Company Research department. Currently, it is an off-line programming. So, it is necessary to return to the programming software to change the settings such as space between two tapes or an angular deviation. Because of the settings duration, the tests are limited. Consequently, if we want to work out an efficiency production line, we have to avoid to return to the programming software. This saves a lot of time. To improve the process, we must find the control strategy most relevant and adjust the accuracy control of the laying-up. In fact, the current system shows a lack of accuracy in some specific cases. The observed results are different according to the relative position between the robot and the mould and the direction of the fibre placement tool (FPT) frame (upwardly, downwardly). Defects as gap and overlap appear. Moreover, because we work in industrial context, the position control loop of the controller is inaccessible for warranty system issues. The control of the robot configuration is seen as a black box. In consequence, the task of laying-up is studied in details. It needs an important compacting strength and a precise fibre location. So, we propose to put in place a sensor-based control scheme with force and vision simultaneously. The process developed must take into account the fact that the internal control loop of robot cannot be modified in the controller to com- 
ply with industrial warranty. In this way, the degrees of freedom are controlled in force, vision or position with an external multi-sensors approach. The fibres placement task is composed of three stages: the approach without contact, the deposit with contact and the withdrawal without contact. The distribution of command (position or/and force or/and vision) is made according to the fibre placement stages. The force control law is already implemented to control the compacting force $\mathbf{F}_{z}$ and the moment $\mathbf{M}_{x}$. The preliminary results are presented in [35]. The visual control law is also implemented separately to control the lateral position and the preliminary results are presented in [34]. So now, we make a multi-sensors approach. It consists of three control loops in parallel with position control loop, force control loop and visual control loop which accumulate the previous approaches. We can see a decrease of disturbing forces and a better location of tape placement along the $y$-axis.

In this paper, a sensor integration is implemented. The results show the improvement of accuracy and the development and experiments are carried out with an industrial prototype of fibre placement. The feedback control has been tested with an experimental test bed. The geometrical structure is identical to that of the industrial robot for fibre placement such that its implementation in industrial environment would be quite easy.

First, the industrial context is explained with the problem presentation and the robot modelling in Sect. 2 . The current system and the industrial specifications are detailed to show the observed problem and to find a new strategy. The kinematic chain and the dynamic model are described to understand the mechatronic system. Then, the sensor-based control is presented in our application case in Sect. 3. The placement tool is controlled in position to forward to the fibre a longitudinal path. A force correction is made along the mould surface normal axis to ensure to the removal tool a good support on the application surface. A visual control is made to offset lateral errors of laying-up in order to avoid the covering and the empty space with the previous tape. Next, the simulation results, the test environment and the experimental results in various configurations are shown in Sect. 4. Afterwards, the discussion is the result balance and the differences and similarities are presented in Sect. 5. Finally, some conclusions and perspectives are offered in Sect. 6.

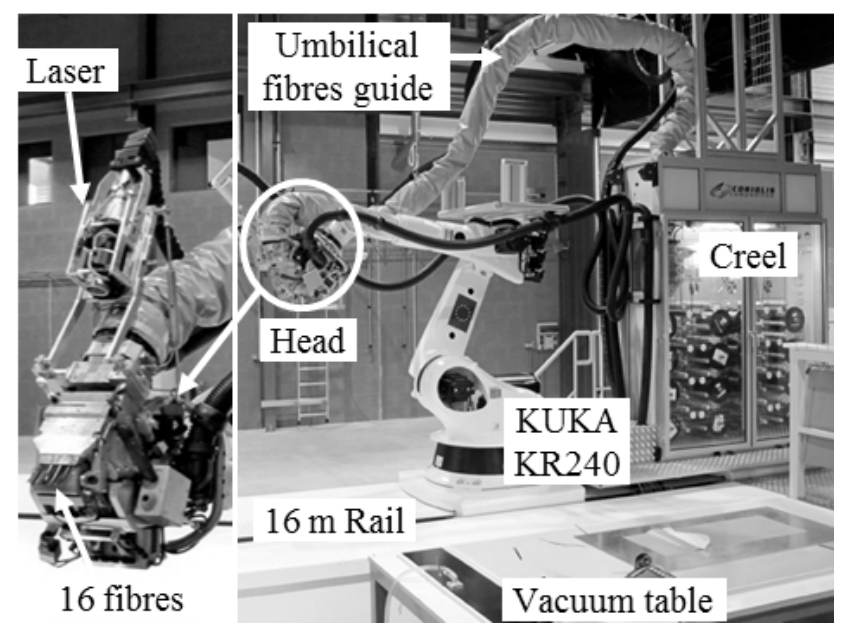

Fig. 1 Robotic cell for Automated Fibre Placement

\section{Manufacturing industrial context}

\subsection{Industrial current system}

\subsubsection{Description}

Coriolis Composites made the engineering of the placement tool using most of industrial components such as a manipulator arm. This new system does not lay-up tape but several fibres depending on the placement tool used. The robotic cell is made of a robot manipulator KUKA KR240 (six joints) with its fibre placement tool developed, see Fig. 1. The robot can be moved on a 16 meters rail (one axis) to work with large pieces. The carbon fibre can be lay-up on two types of support: a vacuum table to lay-up on a fixed horizontal plane and any mould fixed on a positioning system (one axis) allowing to change its orientation. Thus, maximum eight axes have to be synchronised. The carbon fibre coils are located in a creel behind the robot. They are guided to the placement tool thanks to the umbilical fibres guide including flexible pipes developed to avoid fibre damages. The heating system is used to heat the wrapping area. The heating depends on the material and the necessary heating temperatures. An infra-red lamp is used with thermo set which requires temperatures around 50 degrees Celsius. A diode laser is used with dry fibre and thermoplastic. These materials require respectively temperatures around 150-200 degrees Celsius and 350400 degrees Celsius. The carbon fibre width is equal to $6.35 \mathrm{~mm}$. According to the placement tool, it can lay-up from one to eight or 16 or 32 fibres in the same time. Consequently, the width tape varies in accordance to the number of fibres from $6.35 \mathrm{~mm}$ to $50.8 \mathrm{~mm}$ or 101.6 $\mathrm{mm}$ or $203.2 \mathrm{~mm}$. The thickness of one fibre ply varies between $0.1 \mathrm{~mm}$ and $0.3 \mathrm{~mm}$. 
Theoretical tool-path of the placement tool is generated by software CATFiber and CADFiber with CAD data of work-piece and carbon plies orientation. First, the laminate is designed with the number of plies and orientations (Fig. 2-c). Then, plies are created on mould surface based on CAD data. Thus, tapes are generated according to the fibre placement tool used (eight or 16 or 32 fibres). Then, movement strategies have to be chosen: the use of the $16 \mathrm{~m}$ rail to work with large pieces, the modification of positioning system orientation to improve areas access, the lay-up speed and the compacting strength. Finally, tool-paths are created in Kuka Robot Language (KRL). It is a succession of linear movements or spline based displacement. Moreover, the tool-path generation is based only on the geometry of the part, the geometry of the placement tool and the geometric architecture of the robot.

All the directions are controlled in position and orientation thanks to the tool-path creation in KRL language but the following constraints are taken into account by software. Carbon ply has to be compacted and the compacting strength direction must be normal to the surface (Fig. 2-a). The compacting roller is placed at the end of the tool allowing the contact with the material during the lay-up. A pneumatic cylinder is used to manage the compacting strength by adjusting or controlling the pressure inside the cylinder. The tool position along the $x$-axis allows to take into account the displacement speed and the orientation around the $x$-axis allows to spread correctly the compacting force. The tool position along the $y$-axis allows the tape positioning according to the ply definition and the orientation around the $y$ axis is constrained because the $z$-axis must be normal to the mould surface. The tool position along the $z$-axis allows putting in place the tool to activate the pneumatic cylinder and the orientation around the $z$-axis is linked to the tape positioning along the $y$-axis.

\subsubsection{Specifications}

To ensure quality of lay-up, the compacting strength is different according to the material. For dry fibre material, the compacting strength is equal to about $5 \mathrm{~N}$. For thermo plastic material, it is equal to about $200 \mathrm{~N}$ and for thermo set material, it is equal to about $500 \mathrm{~N}$ and it can go up to $1500 \mathrm{~N}$.

According to customer specifications, the laminate can be different, see Fig. 2-b. It is possible to set the distance between tapes called tape interval but also the distance between plies of same orientation called staggering.
Concerning tapes positioning, aeronautical specifications needs important accuracy. The covering between fibres called overlap is forbidden. The spacing unplanned between fibres, called gap, is allowed. For example, with an average width of $300 \mathrm{~mm}$, the cumulative gap should be less than $1.5 \mathrm{~mm}$. The angular deviation should be less than \pm 1 degree .

\subsubsection{Observations}

Some inaccurate fibre placements occur, resulting an inadequate quality of work-piece. In fact, during the lay-up, different defects appear. First, there may be a gap or an overlap between two successive fibres being part of the same tape. Then, there may be a gap or an overlap between two successive tapes regardless of the direction. Finally, the angular deviation may be not fulfilled because the limits are exceeded.

Consequently, we have to identify the source of errors and understand the mechatronic system. The passing of fibres in the re-forwarding device are machined at $6.4 \mathrm{~mm}$. So, the specification sent to the suppliers is that the fibre width must be equal to $6.35 \mathrm{~mm}$. If the fibres width is outside the tolerance, this can bring about a gap or an overlap between fibres being part of the same tape. Thus, a wrong calibration of fibres width leads to defects independently of the robot configuration. Then, a difference between the theoretical fibre tape placement and the practical fibre tape placement may be due to a bad position / orientation of the tool. The robot configuration plays an important role in the appearing of lay-up defaults. Finally, the compacting strength is applied without force feedback to know the real value. As a matter of fact, there is no force sensor on the system. The compacting strength is created by controlling the pressure in the pneumatic cylinder. So, it is based on the relation between the pressure $p$, the force $F$ and the surface $S$ such as $p=\frac{F}{S}$. This hypothesis is valid only if the robot is not deformable under the force (rigid body assumption). It can lead to disturbing forces which generate unwanted displacement of the tool. This is an important element in the fibre placement process.

\subsection{Robot modeling}

\subsubsection{Kinematic chain}

We firstly remind the required material for Automated Fibre Placement. It is a six joints manipulator arm KUKA KR240 with a fibre placement tool. The robot 


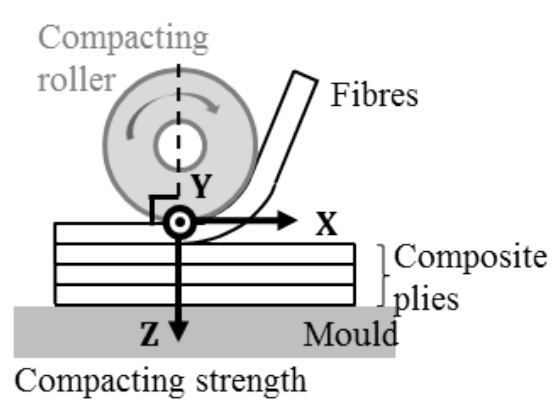

(a)

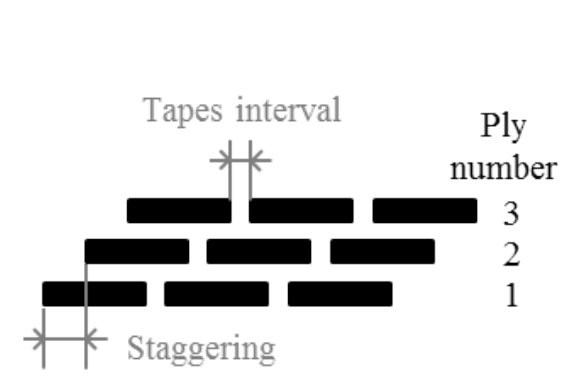

(b)

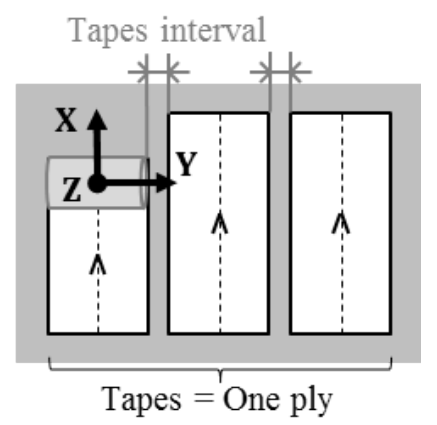

(c)

Fig. 2 Laying-up process: a) Side view; b) Front view; c) Top view

base moves on linear rail. Joints are taken by the $z$ axis. Consequently, rotation is made around $z$-axis and translation is made along $z$-axis. The rotation direction of each joint is defined according to KUKA specifications. To use the Modified Denavit-Hartenberg (MDH) parameters [17], direct frames $(x, y, z)$ are placed respecting the following law for the $x$-axis and $z$-axis: $x_{i}=z_{i+1} \times z_{i}$. The $y$-axis is deduced.

In our context, the kinematic chain is represented in configuration where all joint variables are equal to zero in order to respect the manufacturer choice, see Fig. 3. The world frame axis $F_{w}$ is fixed to the ground. The $z_{w}$-axis is normal to the ground area and guided upwards. The $x_{w}$-axis and the $y_{w}$-axis are in the ground plane. The translation between the frame $F_{1}$ and the frame $F_{0}$ represents the displacement of the robot on the linear rail. The manipulator arm is represented by the frame $F_{1}$ to the frame $F_{7}$. The frame $F_{8}$ corresponds to the force sensor fixed on the wrist of the robot. The frame $F_{12}$ represents the tool end i.e. the compacting roller. Frames are added between $F_{8}$ and $F_{12}$ in order to respect the classic modelling for Modified DenavitHartenberg approach (MDH). Thus, we obtain the table of MDH parameters (Tab. 1) where the geometric parameters $\left(\alpha_{j}, d_{j}, \theta_{j}, r_{j}\right)$ determine the location of the frame $j$ with respect to its antecedent $(j-1)$, see Fig. 4.

\subsubsection{Dynamic model}

The tool of manipulator arm of fibre placement is in contact with a stiff surface through the compacting roller. There is an interaction between the mould and the robot because of compacting strength. Moreover, the umbilical fibres guide disturbs the system. Consequently, we have to take into account this interaction

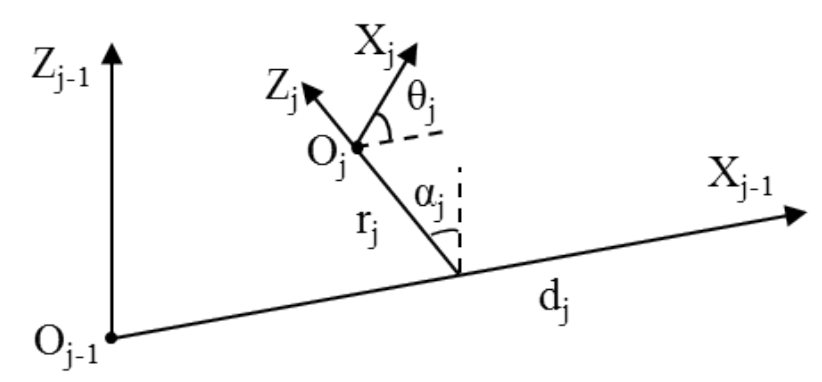

Fig. $4 \mathrm{MDH}$ parameters

Table 1 Modified Denavit-Hartenberg Parameters [17] for Fiber Placement Robot

\begin{tabular}{lllll}
\hline $\mathrm{j}$ & $\alpha_{j}$ & $d_{j}$ & $\theta_{j}$ & $r_{j}$ \\
\hline 1 & 0 & 0 & 0 & $q_{1}$ \\
2 & $\frac{-\pi}{2}$ & 0 & $q_{2}+\frac{\pi}{2}$ & $-r_{2}$ \\
3 & $\frac{-\pi}{2}$ & $-d_{3}$ & $q_{3}$ & 0 \\
4 & 0 & $-d_{4}$ & $q_{4}-\frac{\pi}{2}$ & 0 \\
5 & $\frac{-\pi}{2}$ & $d_{5}$ & $q_{5}+\pi$ & $-r_{5}$ \\
6 & $\frac{-\pi}{2}$ & 0 & $q_{6}+\pi$ & 0 \\
7 & $\frac{-\pi}{2}$ & 0 & $q_{7}$ & 0 \\
8 & $\pi$ & 0 & 0 & $r_{8}$ \\
9 & 0 & 0 & $\frac{\pi}{2}$ & 0 \\
10 & $\alpha_{10}$ & 0 & 0 & 0 \\
11 & 0 & 0 & $\frac{-\pi}{2}$ & 0 \\
12 & 0 & 0 & 0 & $r_{12}$ \\
\hline
\end{tabular}

adding external forces on the classical dynamic model which can be written in the Lagrangian form:

$$
\boldsymbol{\Gamma}+\mathbf{J}^{\top} \mathbf{F}_{e x t}=\mathbf{M}(\mathbf{q}) \ddot{\mathbf{q}}+\mathbf{C}(\mathbf{q}, \dot{\mathbf{q}}) \dot{\mathbf{q}}+\mathbf{G}(\mathbf{q})
$$

$\boldsymbol{\Gamma}$ is the vector of the torques of actuators.

$\mathbf{J}$ is the Jacobian matrix of the tool expressed in the reference frame $F_{0}$.

$\mathbf{F}_{\text {ext }}$ represents the external forces applied on the robot and acts on the tool as $\mathbf{J}^{\top}$ is the Jacobian in the frame $F_{0}$ relative to the tool.

$\mathbf{q}$ is the vector of generalized coordinates. 


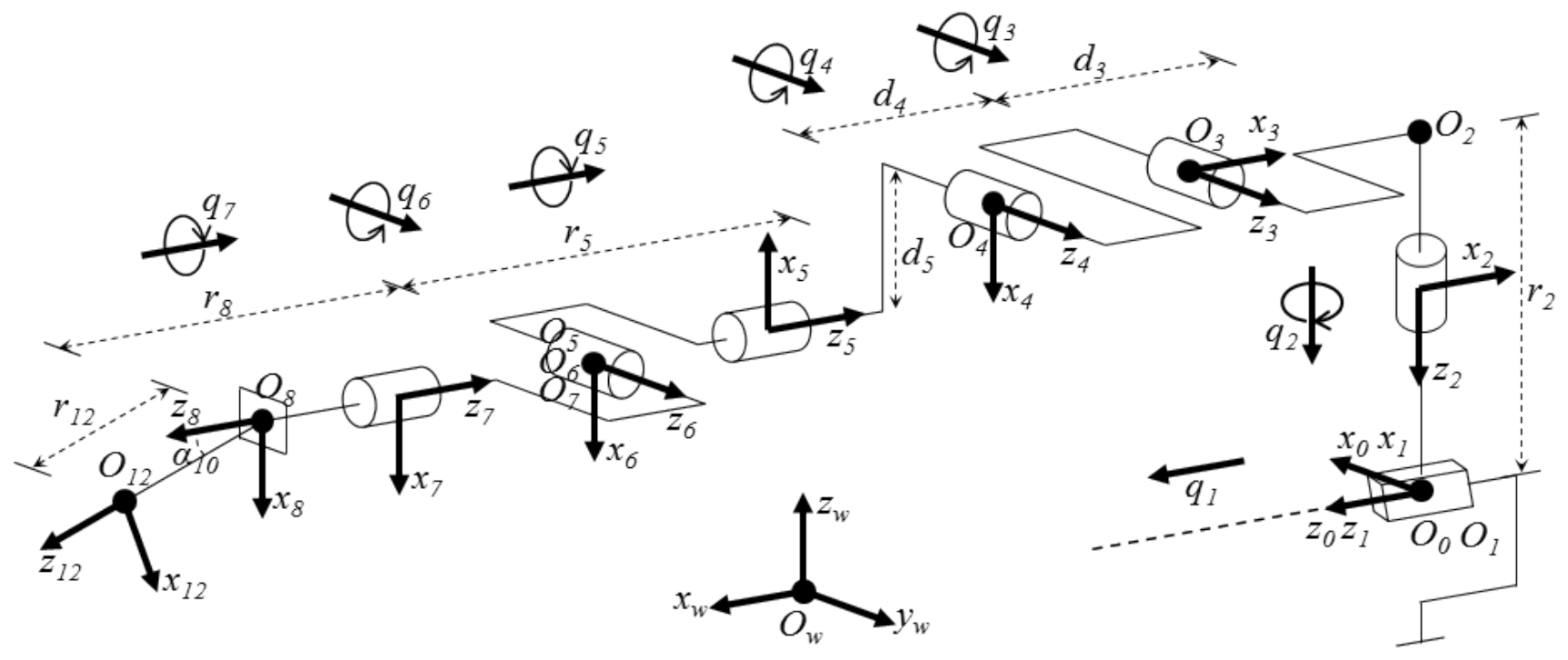

Fig. 3 Geometric architecture

$\mathbf{M}(\mathbf{q})$ is the definite positive inertia matrix.

$\mathbf{C}(\mathbf{q}, \dot{\mathbf{q}})$ is the matrix of the Coriolis and centrifugal effects.

$\mathbf{G}(\mathbf{q})$ is the vector of the gravity effects.

The dynamic model, as presented in Eq. 1, is not implemented in industrial controller. Thus, external force can not be controlled.

\subsubsection{Balance}

To use or change the dynamic model of the system, we need to have access to the robot controller but in our industrial application we cannot modify the robot controller. To take into account the external forces, we have to implement an external sensor-based control.

\subsection{Proposal for enhanced system}

According to the observed problems, the system requires at least a dual command with force / moment and position / orientation. To improve quality of layup, a computer vision based command could be added. Consequently, this paper presents the process of fibre placement with position, force and visual command. We propose to make an interactive manufacturing sensorbased control in the tool coordinate system to take into account the compacting strength and the tape positioning in the new process definition.

\subsubsection{Interactive design}

When create a product or a service, the involved team is firstly making design choices according to technical aspects, like mechanical properties, manufacturing constraints, etc. Secondly, in order to avoid extra costing, the team is looking for feedback from the end users, the maintenance users [23], etc. This is possible only if a draft product is available. To avoid the creation of this prototype, the designers are looking for interactive design which can give the feedback using for example some virtual reality facilities or some design methods mixing simulation and design phases and tools [33].

In [11], the authors report the application of CAD/CAM based technologies for the innovative development of customized surgical devices to assist the mandibular rehabilitation. Design and manufacturing of such customized surgical device are conducted according to the virtual preoperative planning of the surgeon and with the aim to transferring this planning into the operating theater.

In [3], the authors are addressing the machining of a marine propellers which are complex surfaces. The surfaces are usually machined with a perfect roughness not to disturb the theoretical flow (characterized with stream lines) and this requirement is penalizing from a manufacturing point of view. The interactive approach, in this study, is to propose a method in which the machining parameters are linked to functional properties of the blade in order to cancel the polishing phase. To benefit from a rotational axis on a five-axis-machine-tool, the proposed idea is to compose a continuous trajectory, especially at the leading edge, to mill the surface.

In our approach, a GUI has been developed allowing us 
to run some interactive tuning of the setpoint parameters. But the main idea is the interactive manufacturing scheme which permits to run without testing the Offline programming robot trajectories from the CAM software on the workshop facility. The theoretical robot paths are interactively modified in real time by the external sensor based loop.

\subsubsection{Interactive manufacturing setup}

The AFP task is six degrees of freedom task, so the displacement on each axis must be defined. The direction along the $x$-axis must be controlled in position with the curvilinear abscissa to manage the speed displacement of the robot and the orientation around the $x$-axis must be controlled in moment to distribute the compacting strength correctly. The direction along the $y$ axis must be controlled in vision to manage the spacing with the previous tape and the orientation around the $y$-axis must be controlled in position to keep the $z$-axis normal to the surface. The direction along the $z$-axis must be controlled in force to manage the compacting strength and the orientation around the $z$-axis can be controlled in position to manage the tape orientation.

So, a multi-sensors approach is implemented using a force sensor to manage the compacting strength along the $z$-axis and the moment around the $x$-axis, and a visual sensor to manage the tape positioning along the $y$-axis. The others directions are controlled in position. The three commands are active in the same time during the lay-up. In the literature, there are two multisensors approaches: fusion and integration, used to increase accuracy because redundant and complementary information can reduce uncertainties [21]. Sensor integration refers to the control-level combination of sensor feedback whereas sensor fusion refers to the sensorlevel combination [27]. Sensor fusion allows to give some informations about one task direction. The Extended Kalman Filter is used to treat data and estimate the system state $[32,19,13]$. On the contrary, with sensor combination, one sensor gives information on one task direction. Hybrid controller is implemented through diagonal selection matrices which must be orthogonal each other [27]. This allows to assign Cartesian degrees of freedom to each sensor.

In our case, we work with sensor integration because each direction is controlled by only one sensor at the moment. So, selection matrices are used to choose the directions controlled in position, in force and in vision and they must be orthogonal each other i.e. $\mathbf{S}_{p} \perp \mathbf{S}_{f} \perp$ $\mathbf{S}_{v}$. The selection matrices are written considering the

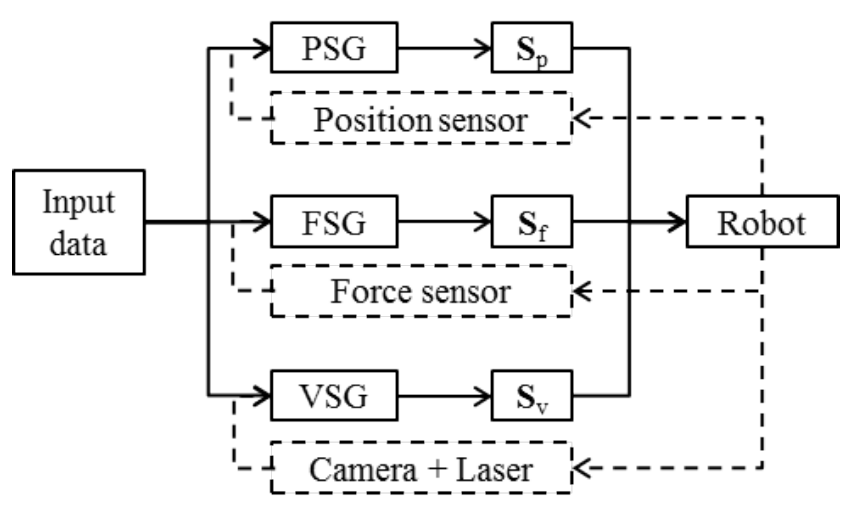

Fig. 5 General control scheme

three positions along $x$-axis, $y$-axis and $z$-axis and the three orientations among $x$-axis, $y$-axis and $z$-axis in the task frame. As a consequence, we have the following selection matrices:

$\mathbf{S}_{p}=\operatorname{diag}(1,0,0,0,1,1)$

$\mathbf{S}_{f}=\operatorname{diag}(0,0,1,1,0,0)$

$\mathbf{S}_{v}=\operatorname{diag}(0,1,0,0,0,0)$

Thanks to the selection matrices, the three commands can be work in the same time. Figure 5 describes the general control scheme with PSG for Position Setpoint Generator, FSG for Force Setpoint Generator and VSG for Visual Setpoint Generator.

\section{Servoing schemes}

\subsection{Position servoing}

Position servoing consist in respecting the position instructions created with tool-path generation. Movements can be joint movement or linear movement according to the stage in the process and the application. In our case, we work with an industrial controller KUKA KR $\mathrm{C} 2$. The controller is considered as a black box because we do not know the exact characteristics (Fig. 6). The implemented model is not explicit and it exists various approaches to control the robot [8]. In most industrial robots, the conventional command is implemented. The mechanism is considered as a linear system and each joint is controlled by a PID command. It exists more advanced methods taking into account the non-linearity of articulated systems for applications requiring high dynamic performances. Here, the internal position loop 


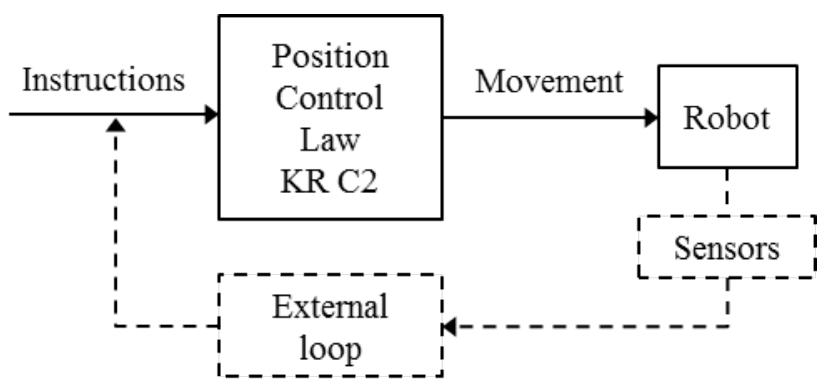

Fig. 6 Position servoing scheme

is inaccessible because it is an industrial application. So, it is not possible to replace the controller for system warranty issues. Consequently, the adding sensor leads the adding of external loop to control the robot. We have to use an overlay to the position control loop to take into account the data from sensors.

\subsection{Force servoing}

In the case of the fibre placement, it is important to control the compacting strength along the $z$-axis named $\mathbf{F}_{z}$ and its distribution corresponding to the moment around the $x$-axis named $\mathbf{M}_{x}$ on the material, Fig. 2 . We use an ATI 6 DOF force/moment sensor to make the measurements. It can measure forces along the $x$-axis, $y$-axis, $z$-axis and moments around the $x$-axis, $y$-axis and $z$-axis. So, we can use the measures of the modulus of $\mathbf{F}_{z}$ and $\mathbf{M}_{x}$ in order to control them and we can observe the others to verify the influence of the correction on the system. The force set-point is very important in this application because it is different according to the material used.

We propose the use of an external hybrid force-position control. The force sensor can be integrated on the system thanks to the Robot Sensor Interface (RSI) developed by KUKA and proposed as an optional component of the KRC2. It is an intermediate system between the inner position control loop implemented in the industrial controller and the external environment. It is an open door allowing us to send command to the inner position control loop. In this way, it is not possible to send directly a program in Kuka Robotics Language (KRL) to the controller. The RSI module can work with various way. It is possible to send it the absolute position desired of the tool in the workshop or the desired joint values. It is also possible to send a tool position increment and the RSI module adds it on the current position. In our configuration, we choose to work with the increment manner of the RSI module because we work with a relative position in the task frame. The task frame formalism is used because there is a contact between tool and work-piece [4]. In this case, each direction of an orthogonal frame related to the tool are separately specified [1]. The task frame is defined by the trajectory generation. As a consequence, there is a constant redefinition between the tool frame and the task frame.

The dual command position - force can be managed in the Cartesian space. In this way, the theoretical toolpath can be followed while applying a controlled compacting strength $\mathbf{F}_{z}$ and a zero moment $\mathbf{M}_{x}$. The movements are computed in the task frame, see Fig. 7. It is important to control the moment $\mathbf{M}_{x}$ because there is a line contact between the compacting roller and the mould surface. So, a moment can appear around the $x$-axis if the orientation is not corrected. This can lead to a bad distribution of the compacting strength on the surface. As a consequence, the measured forces and moments are transformed into the centre of the line contact to have the real forces and moments using the formula of moments transport. First, the wrenches at point A and $\mathrm{B}$ are expressed:

$\tau_{A}=\left[\begin{array}{c}\mathbf{F}_{\mathbf{A}} \\ \mathbf{M}_{\mathbf{A}}\end{array}\right]_{A}=\left[\begin{array}{c}\mathbf{F}_{\mathbf{A}} \\ \mathbf{M}_{\mathbf{A}}+\mathbf{B A} \times \mathbf{F}_{\mathbf{A}}\end{array}\right]_{B}$

$\tau_{B}=\left[\begin{array}{c}\mathbf{F}_{\mathbf{B}} \\ \mathbf{M}_{\mathbf{B}}\end{array}\right]_{B}$

If the Fundamental Principle of Static is applied, we obtain:

$\left[\begin{array}{c}\mathbf{F}_{\mathbf{A}} \\ \mathbf{M}_{\mathbf{A}}+\mathbf{B A} \times \mathbf{F}_{\mathbf{A}}\end{array}\right]_{B}+\left[\begin{array}{l}\mathbf{F}_{\mathbf{B}} \\ \mathbf{M}_{\mathbf{B}}\end{array}\right]_{B}=\left[\begin{array}{l}\mathbf{0} \\ \mathbf{0}\end{array}\right]_{B}$

So, we have the following equalities:

$\mathbf{F}_{\mathbf{A}}+\mathbf{F}_{\mathbf{B}}=\mathbf{0}$

$\mathbf{M}_{\mathbf{A}}+\mathbf{B A} \times \mathbf{F}_{\mathbf{A}}+\mathbf{M}_{\mathbf{B}}=\mathbf{0}$

Finally, we obtain the following result:

$\mathbf{F}_{\mathrm{B}}=-\mathbf{F}_{\mathbf{A}}$

$\mathbf{M}_{\mathbf{B}}=-\left(\mathbf{M}_{\mathbf{A}}+\mathbf{B A} \times \mathbf{F}_{\mathbf{A}}\right)$

Consequently, the wrench can be written:

$\tau_{B}=\left[\begin{array}{c}\mathbf{F}_{\mathbf{B}} \\ \mathbf{M}_{\mathbf{B}}\end{array}\right]_{B}=-\left[\begin{array}{c}\mathbf{F}_{\mathbf{A}} \\ \mathbf{M}_{\mathbf{A}}+\mathbf{B A} \times \mathbf{F}_{\mathbf{A}}\end{array}\right]_{B}$

The external loop computes the increment in millimetres and degrees that the FPT must reach corresponding to the errors in force and moment computed from the measurements using (8). In this way, the increment on the $z$-direction and the increment around the $x$ orientation are computed according to the force and moment errors thanks to the force control law. The 


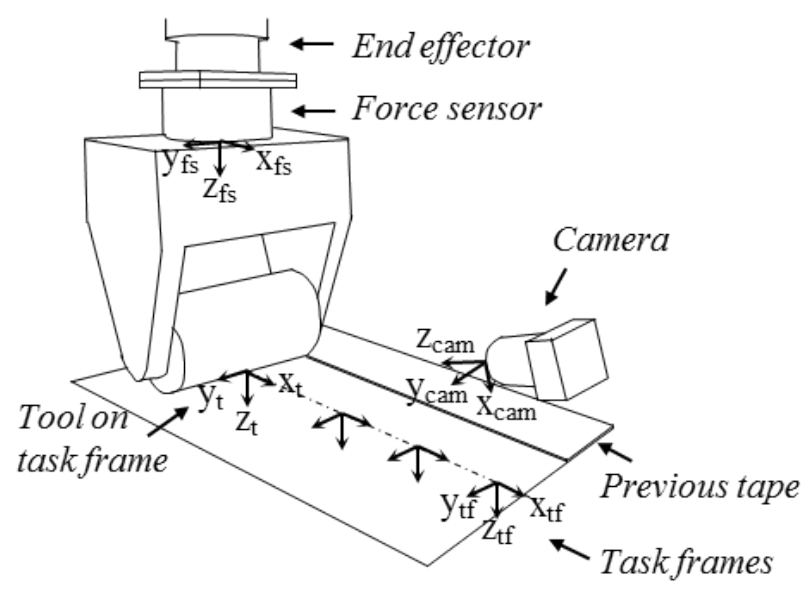

Fig. 7 Task frame and transformation of moment

force control law is described in Sec. 3.5. The variables $e(k), e(k-1)$ and $e(k-2)$ represent the errors in newton along the $z$-axis in the task frame.

During the process, there are different stages. First, the tool goes down on the mould and a position control is used because the compacting roller is not in contact with the laying surface (no force control). Secondly, the tool goes down in contact with the material, so the compacting strength and its distribution must be controlled. Then, the tool rolls and applies the fibres on the mould and the compacting strength must be constant. Finally, the tool goes up and the position control is sufficient. To choose when and what directions are controlled in force or position, diagonal selection matrices $\mathbf{S}_{p}$ and $\mathbf{S}_{f}$ are used. If there is no desired force, the selection matrix $\mathbf{S}_{f}$ does not select the force command along the $z$-axis and the system is controlled in position.

Figure 8, we can see the mechanism of the external hybrid position-force control. First, the desired wrench $\mathbf{F}_{\text {des }}$ is compared to the wrench measured $\mathbf{F}_{\text {meas }}$. The error in force $\Delta \mathbf{F}$ must be converted in error of position $\Delta \mathbf{X}_{F}$ through the force control law. Simultaneously, the position-orientation desired $\mathbf{X}_{d e s}$ is compared to the position-orientation measured $\mathbf{X}$. The error $\Delta \mathbf{X}$ is converted in $\Delta \mathbf{X}_{P}$ through the position control law. Then, the error of position $\Delta \mathbf{X}_{F P}$ is composed of directions controlled in force, and other directions controlled in position thanks to the selection matrix, and is sent to the RSI module. This one is added to the current position and is sent to the inner position control loop.

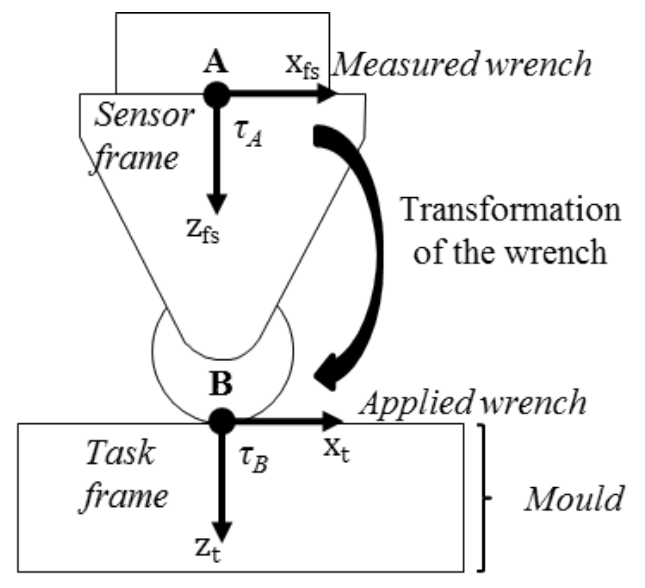

\subsection{Visual servoing}

Because the gap must be managed and the overlap must be avoided, the lateral position of the FPT is important. The lateral position corresponds to the $y$-direction and it is controlled with a visual control scheme. So it is named $V_{y}$. In the industrial system, the position of each tape is separately defined before the lay-up in off-line programming. Without correction during the lay-up, a difference between the theoretical and the real tool-path can appear. Consequently, this difference can lead to positioning errors. In this idea, a visual control scheme is implemented along the $y$-axis to respect the interval between two tapes, Fig. 2. The objective is to control the lay-up of the new tape with respect to the previous tape edge in order to make on-line correction. To do this, a high speed camera coupled with a laser projector is used. The laser line is projected on the surface of the part and it is perpendicular to the ply in progress. The edge of the previous tape creates a deformation of the laser line observed by the camera, Fig. 9. The thickness variation is detected and its location is measured in pixel on the image grabbed from the camera after processing. The desired location of the previous tape edge (to obtain the desired gap between tapes) is defined in pixels coordinates in the image frame. To do so, the active vision system needs to be calibrated. The row information of the defined target is relevant for the visual servoing. Then, the location of the previous tape edge is compared with the target to compute the error in pixel. The camera is positioned and oriented such as the line on the image is along the $y$-axis of the tool, Fig. 7. In this way, the FPT has to move along the $y$ axis in order to correct this error.

To command the displacement of the FPT using the visual servoing along the $y$-axis, an external loop is 


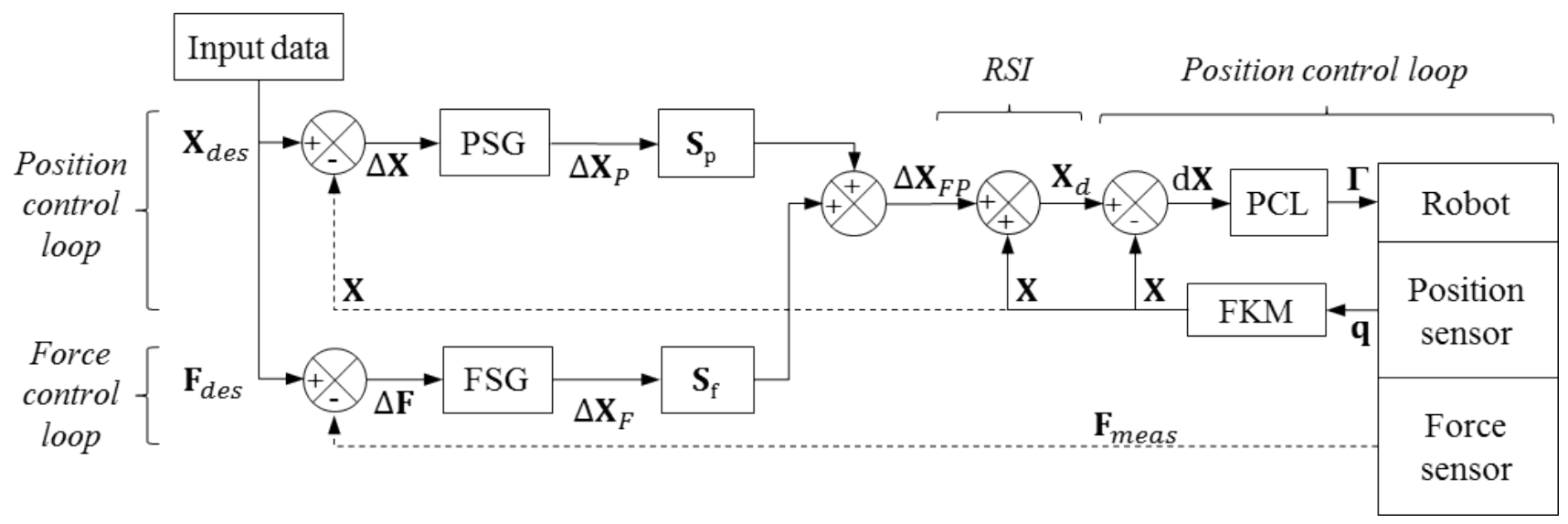

Fig. 8 Force Servoing Scheme

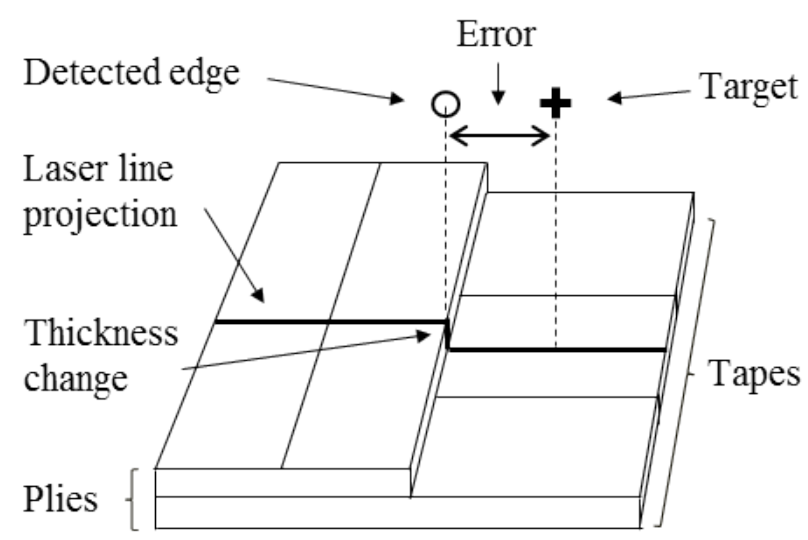

Fig. 9 Thickness detection

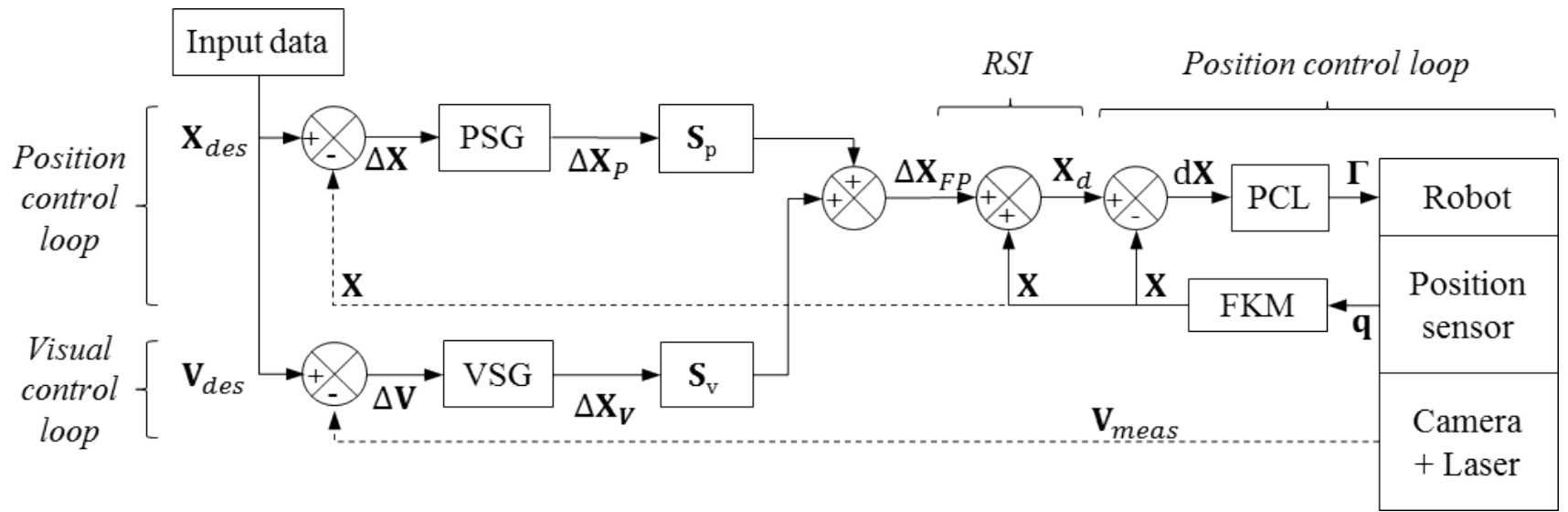

Fig. 10 Visual servoing scheme

added on the initial system in the same manner as the force servoing. The RSI module is used as an intermediary between the vision system and the robot. The dual command position-vision can be managed in the Cartesian space. In this way, the theoretical tool-path can be followed while a correction is made along the $y$-axis to respect the tapes interval.
The external loop computes the displacement in millimetres that the FPT must perform in order to correct the error measured in pixel on the image grabbed from the camera. The displacement along the $y$-direction is computed following the alignment errors thanks to the visual control law. A PID controller is implemented on the visual control law in the same manner as the force control law. Here, the variables $e(k), e(k-1)$ and 
$e(k-2)$ represent the errors in pixel along the $y$-axis in the image. This control law is described in Sec. 3.5.

To test the visual servoing, there are different stages. First stage, the tool goes down at the approach position above the mould using the position control law. So, there is only one selection matrix used, named $\mathbf{S}_{p}$. Second stage, the visual control law allows correcting the location of the FPT along the $y$-axis using the selection matrix $\mathbf{S}_{v}$. Then in third stage, the FPT can make displacement along the $x$-axis while maintaining the correct tapes interval thanks to the visual control law. Here, the displacement is made without contact in order to not disturb the visual control.

Figure 10, we can see the mechanism of the external hybrid position-visual control. First, the location desired of the tape edge into the image $\mathbf{V}_{\text {des }}$ is compared to the location measured into the image $\mathbf{V}_{\text {meas }}$. The error in pixel $\Delta \mathbf{V}$ must be converted in error of position $\Delta \mathbf{X}_{V}$ through the visual control law. Simultaneously, the position-orientation desired of the FPT $\mathbf{X}_{\text {des }}$ is compared to the position-orientation measured $\mathbf{X}$. The error $\Delta \mathbf{X}$ is converted in $\Delta \mathbf{X}_{P}$ through the position control law. Then, the error of position $\Delta \mathbf{X}_{V P}$ is composed of one direction controlled in vision, and other directions controlled in position thanks to the selection matrix, and is sent to the RSI module. This one is added to the current position and it is sent to the inner position control loop.

\subsection{Position-Force-Visual servoing}

In the sections 3.2 and 3.3 , the force servoing and the visual servoing are separately implemented. Those are two independent tests. In the first case, we study the benefits of controlling the compacting strength along the $z$-axis and the moment around the $x$-axis thanks to a force control scheme. In the second case, we study the benefits of controlling the lateral position along the $y$ axis thanks to a visual control scheme. Here, we put in parallel the three types of servoing: position, force and visual using a multi-sensors approach. The objective is to merge the previous approaches to have a complete system.

To choose which directions are controlled in position, force or vision, the selection matrices are modified following the steps of the lay-up, Tab. 2. In the step 1, the FPT goes down to the approach position and all the directions are controlled in position. In the step 2, the FPT location along the $y$-axis is corrected with respect to the previous tape if it is necessary. Here, the $y$-direction is controlled in vision and the other directions are controlled in position. In the step 3, the FPT goes down to have the desired compacting strength and a correct distribution of the force on the surface while keeping the correct lateral location with respect to the previous tape. Here, the $y$-direction is controlled in vision, the $z$-direction is controlled in force, the $x$ direction is controlled in moment and the other directions are controlled in position. In the step 4, the FPT moves along the $x$-direction to lay-up a tape while applying the desired compacting force with a distribution of it and a correct lateral correction. So, the directions are controlled in the same manner as the step 3 . In the step 5, the FPT goes up to the departure position. All the directions are controlled in position.

Figure 11, we can see the mechanism of the sensors integration with three external loops which integrate the position control loop, the force control loop and the visual control loop. According to the selection matrices $\mathbf{S}_{p}, \mathbf{S}_{f}$ and $\mathbf{S}_{v}$, the increment of position $\Delta \mathbf{X}_{F V P}$ is computed from the position servoing or/and the force servoing or/and the visual servoing. This control scheme is the addition of the force control scheme (Fig. 8) and the visual control scheme (Fig. 10).

\subsection{Setpoint generator}

Regulation can be done with a numerical PID because we work with an external loop and our system is already controlled in closed loop (industrial controller). The controller output for a continuous time expression of a PID controller is written below:

$u(t)=K_{p}\left[e(t)+\frac{1}{T_{i}} \int_{0}^{t} e(\tau) \mathrm{d} \tau+T_{d} \frac{\mathrm{d} e(t)}{\mathrm{d} t}\right]$

The integral term of the equation (9) is approximated by a finite sum where $\Delta$ is the sampling period:

$$
\sum_{i=1}^{k} e(i) \Delta \quad ; \quad t=k \Delta
$$

The first order derivative term of the equation (9) is approximated by the backward finite difference:

$\frac{e(k)-e(k-1)}{\Delta}$

Replacing the integral term (10) and the derivative term (11) in the equation (9), one can obtain:

$u(k)=K_{p}\left[e(k)+\frac{\Delta}{T_{i}} \sum_{i=1}^{k} e(i)+\frac{T_{d}}{\Delta}(e(k)-e(k-1))\right]$ 
Table 2 Selection matrices following the steps of lay-up

\begin{tabular}{cccccc}
\hline Step & 1 & 2 & 3 & 4 & 5 \\
\hline $\mathbf{S}_{p}$ & {$[1,1,1,1,1,1]$} & {$[1,0,1,1,1,1]$} & {$[1,0,0,0,1,1]$} & {$[1,0,0,0,1,1]$} & {$[1,1,1,1,1,1]$} \\
$\mathbf{S}_{f}$ & {$[0,0,0,0,0,0]$} & {$[0,0,0,0,0,0]$} & {$[0,0,1,1,0,0]$} & {$[0,0,1,1,0,0]$} & {$[0,0,0,0,0,0]$} \\
$\mathbf{S}_{v}$ & {$[0,0,0,0,0,0]$} & {$[0,1,0,0,0,0]$} & {$[0,1,0,0,0,0]$} & {$[0,1,0,0,0,0]$} & {$[0,0,0,0,0,0]$} \\
\hline
\end{tabular}

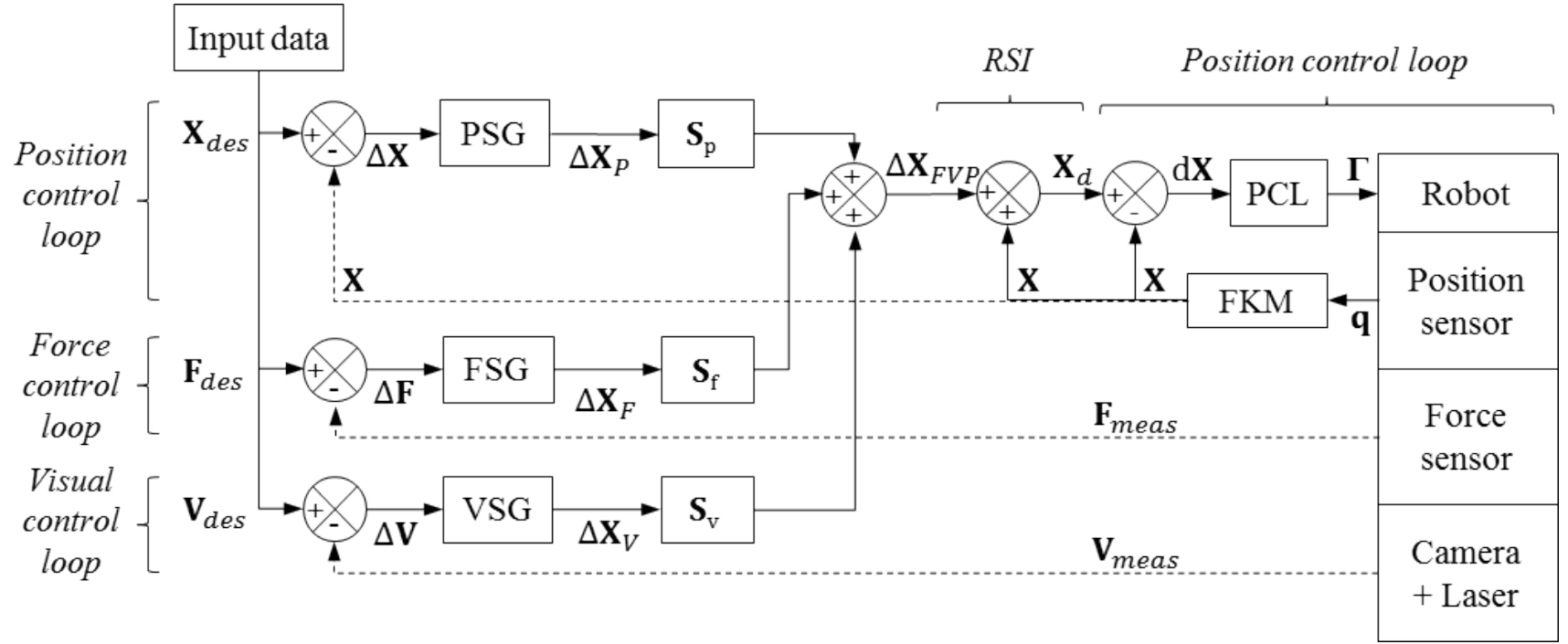

Fig. 11 Position-force-visual servoing scheme

(12) $e(k-2)$ which are the errors at the instants $k \Delta,(k-$ $1) \Delta$ and $(k-2) \Delta$. The parameters $K_{0}, K_{1}$ and $K_{2}$ can

The error values at some previous time instants have to be stored. To have $\Delta u(k), u(k-1)$ is subtracted from $u(k)$ and one can obtain:

$\Delta u(k)=u(k)-u(k-1)$

$$
\begin{aligned}
\Delta u(k)=K_{p}[e(k) & -e(k-1)+\frac{\Delta}{T_{i}} e(k) \\
& \left.+\frac{T_{d}}{\Delta}(e(k)-2 e(k-1)+e(k-2))\right]
\end{aligned}
$$

$\Delta u(k)=K_{0} \cdot e(k)+K_{1} \cdot e(k-1)+K_{2} \cdot e(k-2)$

where :

$K_{0}=K_{p}\left(1+\frac{\Delta}{T_{i}}+\frac{T_{d}}{\Delta}\right)$

$K_{1}=-K_{p}\left(1+\frac{2 T_{d}}{\Delta}\right)$

$K_{2}=K_{p}\left(\frac{T_{d}}{\Delta}\right)$

At each sample instant, the incremental output of position $\Delta u(k)$ is computed and sent to the RSI module because we work in relative position in the tool space. Three previous values are necessary: $e(k), e(k-1)$ and be viewed as tuning parameters. Knowing that $T_{i}=\frac{K_{p}}{K_{i}}$ and $T_{d}=\frac{K_{d}}{K_{p}}$, the tuning parameters can be set from $K_{p}, K_{i}$ and $K_{d}$ such as:

$K_{0}=K_{p}+K_{i} \Delta+\frac{K_{d}}{\Delta}$

$K_{1}=-K_{p}-\frac{2 K_{d}}{\Delta}$

$K_{2}=\frac{K_{d}}{\Delta}$

This incremental formulation can only be used to implement only PID controller (no PI or PD controller) because the original parameters $K_{p}, K_{i}$ and $K_{d}$ take part into the $K_{0}, K_{1}$ and $K_{2}$ parameters.

\section{Results}

In this part, we firstly present the experimental environment, its design and optimization and finally the experimental result of fibre placement laying-up process using our proposed control schemes:

- Position - force servoing,

- Position - visual servoing,

- Position - force - visual servoing. 


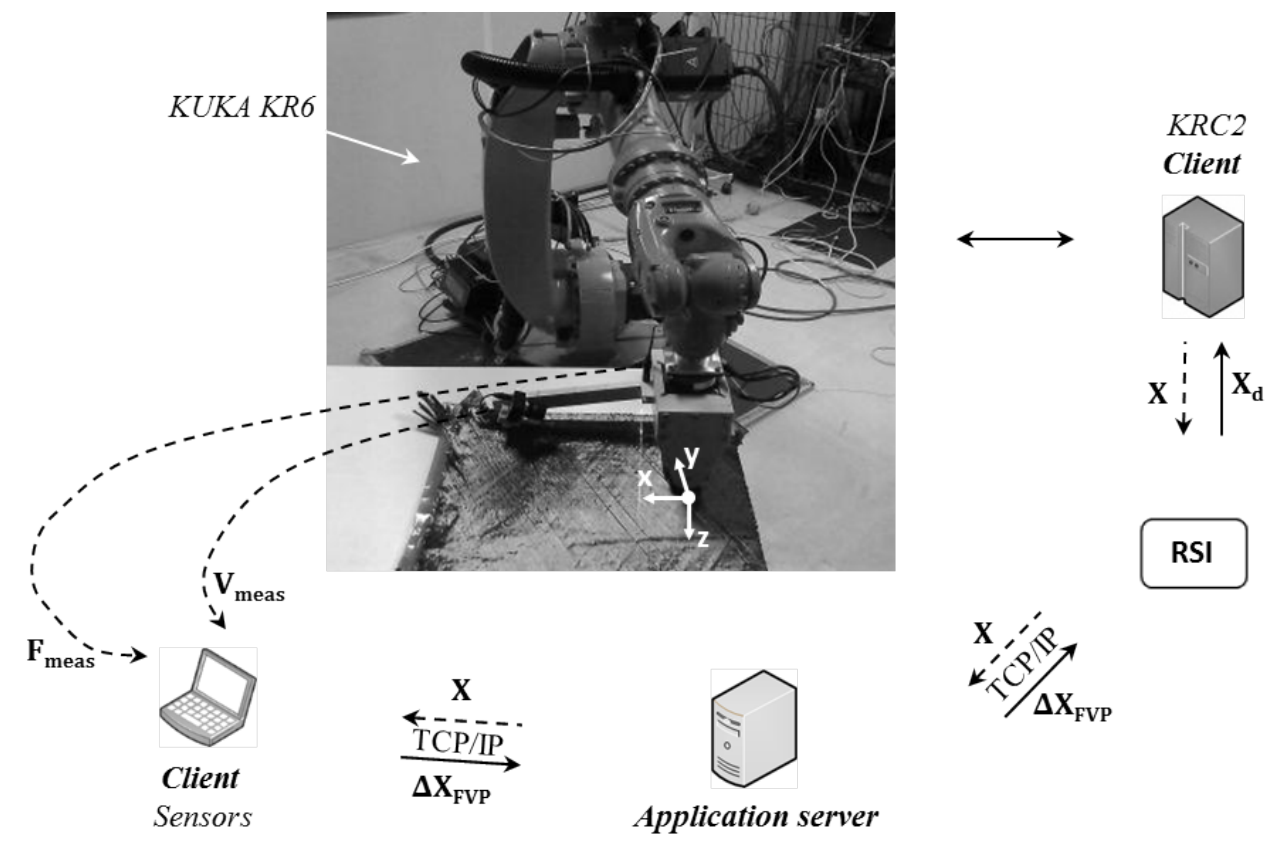

Fig. 12 Test environment

4.1 Experimental environment

\subsubsection{Hardware devices}

For testing, the robotic cell of the research laboratory is used. It consists of a KUKA KR6 manipulator arm (Fig. 12). This robot is smaller than the industrial robot KUKA KR240 used for the Automated Fibre Placement. In fact, dimensional and inertial parameters change but the robots have the same geometric architecture. Moreover, both have the same controller KRC2. This allows to work with the Robot Sensor Interface (RSI) module which is an intermediary system between the robot and the sensors. It must receive data in a sampling period of $12 \mathrm{~ms}$, otherwise data is lost and if too many data are lost then the KRC2 controller takes back the control of the arm for security purposes.

To simulate the process on our experimental setup, an effector is made in order to emulate the FPT and fixed on the manipulator arm (Fig. 12). A compacting roller, taken from the real industrial machine, is added on the support. However, there is no fibre in this system to lay-up. Measurement of forces and moments must be as close to the compacting roller. Consequently, the force sensor is attached on the wrist of the robot between the FPT and the manipulator arm. Then, the laser is fixed on the effector and it projects a line on the part surface. The camera is placed on the effector via the intermediary of a fixing arm allowing the distance and an orientation between the laser and the camera to be tuned.

\subsubsection{Software application}

A client-server type communication is developed between elements. In this way, the client robot can communicate with the client sensors thanks to the application server via a TCP/IP communication. The robot send its current position $\mathbf{X}$ to the RSI module. It transmits it to the server and then, it is sent to the client "sensors". In the same time, the client sensors receives data from the force sensor $\mathbf{F}_{\text {meas }}$ and the camera $\mathbf{V}_{\text {meas }}$. It computes the correction for the FPT to do in millimetres $\Delta \mathbf{X}_{F V P}$ and sends it to the application server which transmits it to the RSI module. The RSI module adds the correction computed to the current position and it sends it $\mathbf{X}_{d}$ to the robot.

The sensor client application has been developed using C sharp programming language, Visual Studio IDE and emguCV (a C sharp class wrapper for OpenCV Image processing library). These choices allow us a fast and powerful software development by using existing classes for TCP/IP connexion, for the graphical user interface (GUI) development, chart design for live data plotting... A GUI snapshot is presented in Fig. 13. It is composed of seven parts. The area A allows the connection to the camera. It shows edge detection in pixel and it allows controlling the $y$-direction with visual control law. If the $y$-direction is controlled with visual servoing, 


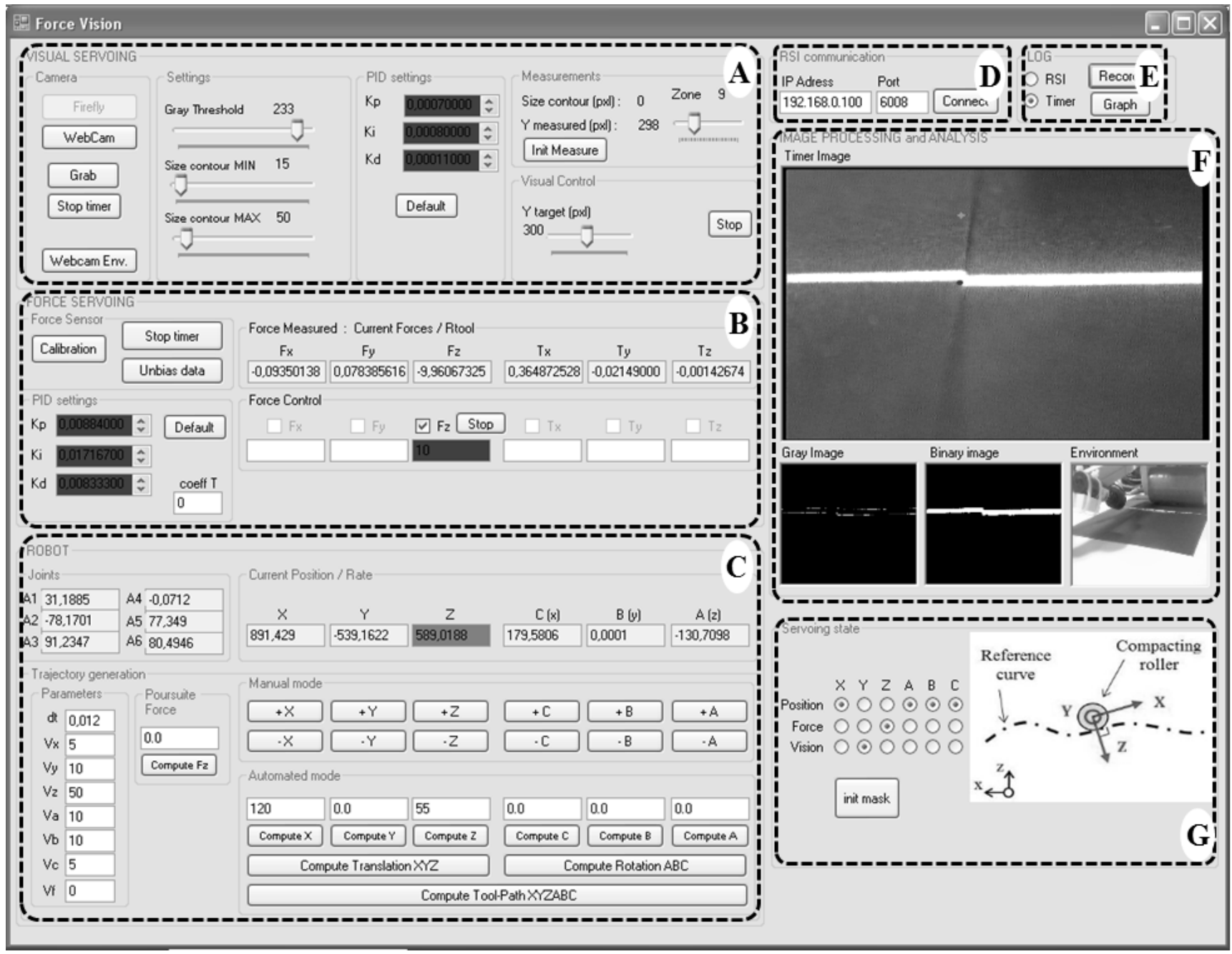

Fig. 13 GUI of the application

it cannot be controlled with position or force servoing because of the selection matrix. The area B allows the force sensor calibration and doing a bias of data to remove the gravity effect. It shows force and torque measurements. It also allows choosing which direction is force controlled. In accordance with the selection matrix, the direction controlled in force cannot be controlled in position. The area $\mathrm{C}$ shows the joint values of the robot and the Cartesian values of the tool in the reference frame. It also manages the trajectory generation by linear movement for RSI module. The area D allows connecting to the server in order to communicate with the RSI module. The area E allows recording data in text file that can be opened in MATLABß for analysis and display. Data is composed of position-orientation of the tool in reference frame, the wrench measures and the lateral position in pixel. The area F shows the image processing and analysis and the environment. The area $\mathrm{G}$ shows which direction is controlled in position, force or vision. The live variables such as desired force, measured force, robot position and edge detection in the image could be displayed if needed (not shown in picture).

\subsection{Active vision system}

As the thickness of the fibre is really low $(0.1 \mathrm{~mm}$ to 0.3 $\mathrm{mm}$ ), the relative positioning of the triplet: laser source, camera and compacting roller needs to be studied before the engineering of the experiment setup parts. To do so, we have setup a $3 \mathrm{D}$ simulation environment programmed under MATLAB to optimize the edge detection. Figure 14 presents a view of our 3D simulator showing the FPT, the laser beam, the intersections between laser plane and part surface and the camera frame at several locations. 


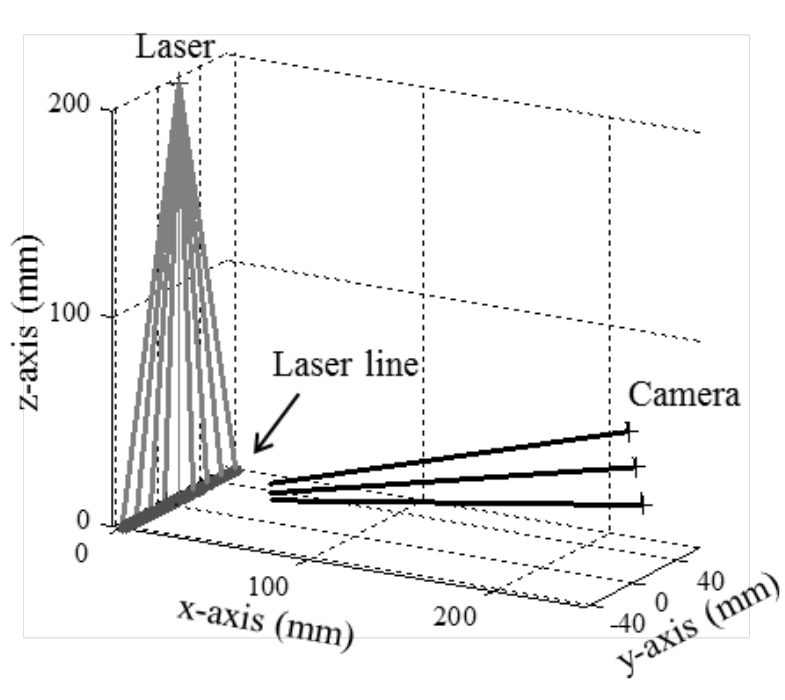

Fig. 14 View of the 3D simulator

\subsubsection{Camera modeling and calibration}

To choose the camera resolution, its position and orientation with respect to compaction roller and laser beam, we have to model the camera to be able to run a $3 \mathrm{D}$ simulation.

\section{Modeling}

We use a classical pinhole camera model [2]. In this model, a scene view is formed by projecting $3 \mathrm{D}$ points into the image plane using a perspective transformation.

$s\left[\begin{array}{l}u \\ v \\ 1\end{array}\right]=\left[\begin{array}{ccc}f_{x} & 0 & c_{x} \\ 0 & f_{y} & c_{y} \\ 0 & 0 & 1\end{array}\right]\left[\begin{array}{llll}r_{11} & r_{12} & r_{13} & t_{1} \\ r_{21} & r_{22} & r_{23} & t_{2} \\ r_{31} & r_{32} & r_{33} & t_{3}\end{array}\right]\left[\begin{array}{c}X \\ Y \\ Z \\ 1\end{array}\right]$

where:

- $s$ is the scaling factor applied to the image

- $(X, Y, Z)$ are the coordinates of a $3 \mathrm{D}$ point of the

laser stripe in the world coordinate space

- $[R \mid t]$ is the matrix of extrinsic parameters

- $(u, v)$ are the coordinates of the projection point in pixels

- $\left(c_{x}, c_{y}\right)$ is a principal point that is usually at the image centre

- $f_{x}, f_{y}$ are the focal lengths expressed in pixel units

The matrix of intrinsic parameters does not depend on the scene viewed. So, once estimated, it can be re-used as long as the focal length is fixed (in case of zoom lens). The joint rotation-translation matrix $[R \mid t]$ is used to describe the camera position and orientation. That is, $[R \mid t]$ translates coordinates of a point $(X, Y, Z)$ to a coordinate system, fixed with respect to the camera. The transformation above is equivalent to the following (when $z \neq 0$ ):

$$
\begin{aligned}
& {\left[\begin{array}{l}
x \\
y \\
z \\
1
\end{array}\right]=R\left[\begin{array}{l}
X \\
Y \\
Z \\
1
\end{array}\right]+t} \\
& x^{\prime}=\frac{x}{z} \\
& y^{\prime}=\frac{y}{z} \\
& u=f_{x} \times x^{\prime}+c_{x} \\
& v=f_{y} \times y^{\prime}+c_{y}
\end{aligned}
$$

Real lenses usually have some distortion, mostly radial distortion and slight tangential distortion, the above model can be extended to include distortion modelling. While the distortion coefficients are the same regardless of the camera resolutions used, the focal lengths and image centre should be scaled along with the current resolution from the calibrated resolution (calibrated at $640 \times 480$ and used at $320 \times 240$ ).

\section{Calibration}

With calibration, we determine the relation between the camera's natural units (pixels) and the real world units (for example millimetres). This is useful when we want to determine the best location and orientation for a given size target to observe (fibre thickness in our case). The unknown parameters are $f_{x}, f_{y}$ and $\left(c_{x}, c_{y}\right)$ and expressed in pixels coordinates. If for both axes a common focal length is used with a given $a$ aspect ratio (usually 1 ), then $f_{y}=f_{x} \times a$ and in the upper formula we will have a single focal length $f$. The process of determining these two matrices is the calibration. Calculation of these parameters is done through basic geometrical equations. The equations used depend on the chosen calibrating objects. We have chosen to use the OpenCV image processing library which supports three types of objects for calibration:

- Classical black-white chessboard

- Symmetrical circle pattern

- Asymmetrical circle pattern.

Basically, we take snapshots of the chosen pattern with the camera and let OpenCV function find the estimated parameters. Each found pattern results in a new equation. To solve the equation, we need at least a predetermined number of pattern snapshots to form a wellposed equation system. In practice, we have a noise present in our input images, so for good results, we need at least 10 good snapshots of the input pattern in different positions [2]. 


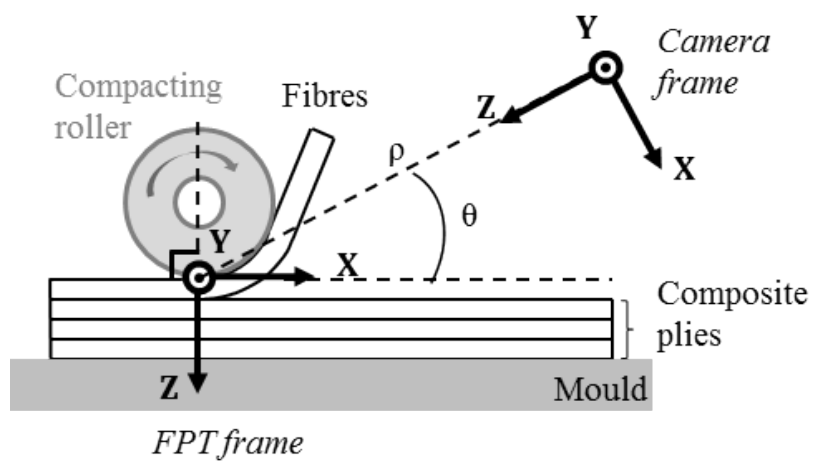

Fig. 15 Active vision frames description

\subsubsection{Joint rotation-translation matrix}

The matrix of extrinsic parameters $[R \mid t]$ translates coordinates of a point $(X, Y, Z)$ in the $3 \mathrm{D}$ world coordinate system to the camera coordinate system. In our case, we have chosen to describe this matrix with a rotation around $Y$-axis of the FPT to have the camera axis pointing toward the tool centre point of the FPT and a distance in polar coordinates $[\rho, \theta]$ (see Fig. 15). The extrinsic matrix is defined as follow:

$M_{\text {extrinsic }}=\left[\begin{array}{ccccc}\cos \left(\theta-\frac{\pi}{2}\right) & 0 & -\sin \left(\theta-\frac{\pi}{2}\right) & \rho \cos (\theta) \\ 0 & 1 & 0 & P_{y} \\ \sin \left(\theta-\frac{\pi}{2}\right) & 0 & \cos \left(\theta-\frac{\pi}{2}\right) & \rho \sin (\theta) \\ 0 & 0 & 0 & 1\end{array}\right]$

\subsubsection{Extrinsic matrix optimization}

According to a given camera (intrinsic parameters), we optimize the laser edge detection by tuning the $[\rho, \theta]$ parameters, Fig. 16. We also need to take into account the geometric constraints on the FPT so the camera location should not interfere with the laying up environment and especially the mould surface. The chosen values for $[\rho, \theta]$ are the following:

$200 \mathrm{~mm}<\rho<280 \mathrm{~mm}$

$$
8^{\circ}<\theta<10^{\circ}
$$

Figure 17 shows the experimental setup we are using for the experiments.

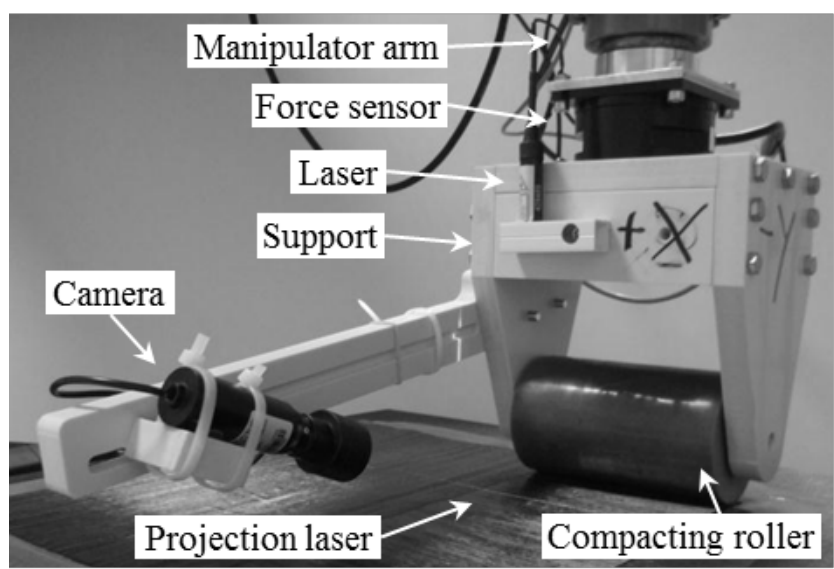

Fig. 17 Camera, laser beam setup on the FPT

\subsection{Experimental results}

\subsubsection{Position - Force servoing}

\section{Force servoing}

We present here some results of force control among the $z$-axis without displacement on the five other directions. The aim of this experiment is to validate the PID parameters for the FCL of the hybrid control scheme. We have run two types of tests:

- step input (0 N to $20 \mathrm{~N})$,

- slope input (10 N to $40 \mathrm{~N})$.

In Fig. 18-a, we can see that the $z$-displacement starts when the force step input is activated (at $t=1600 \mathrm{~ms}$ ). The first part of this displacement does not make any changes on the measured force value because the compaction roller is not in contact with the mould surface. Then (at $t=3300 \mathrm{~ms}$ ), the contact force appears and the displacement continues to reach the target value of 20 N. A little over shoot of $1.64 \mathrm{~N}(+8 \%)$ is observed and then the measured force reached the target value. We can notice that the final error is very small (less than $\pm 2 \%)$.

In Fig. 18-b, we present the results for the slope input. Before the slope, the force is regulated to $10 \mathrm{~N}$ which is the start value of the slope input. The $z$-displacement has also slope profile but we can see the non linearity of the compaction roller stiffness. The system response can be split in two parts:

- from $2428 \mathrm{~ms}$ to $4442 \mathrm{~ms}$,

- from $4442 \mathrm{~ms}$ to $31530 \mathrm{~ms}$.

In the first part, we can notice that the following error (lag) is very small (less than $\pm 5 \%$ ) and it takes $2014 \mathrm{~ms}$ to reach the setpoint. In the second part, the average 

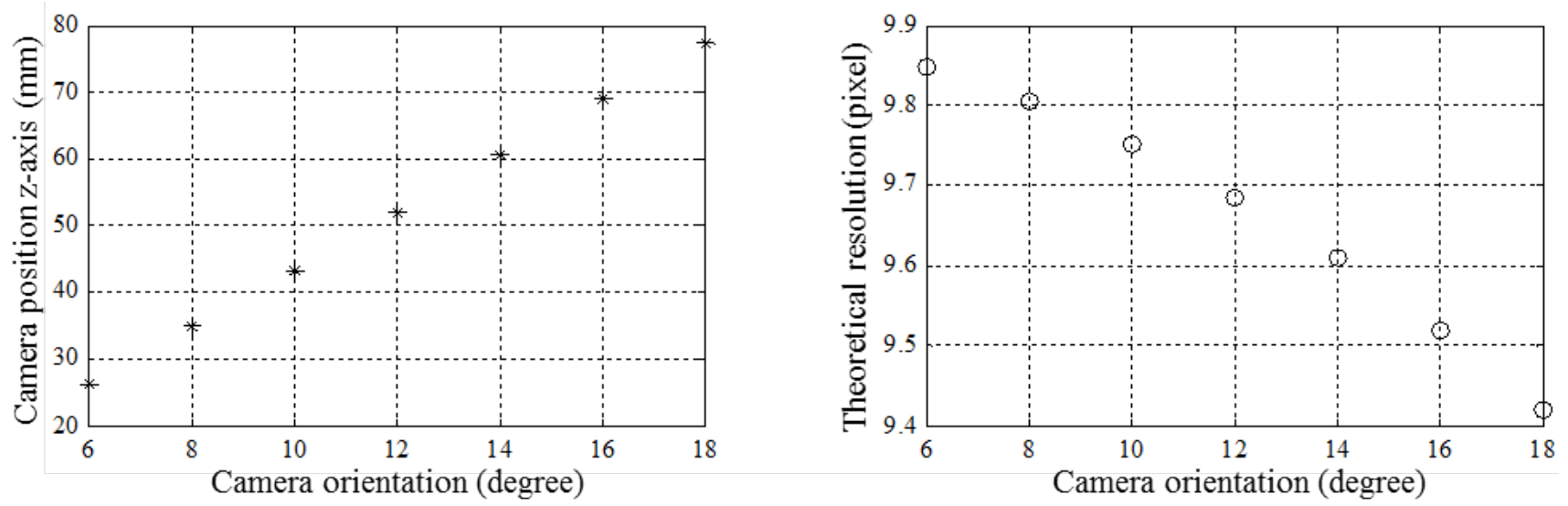

Fig. 16 Active vision optimization

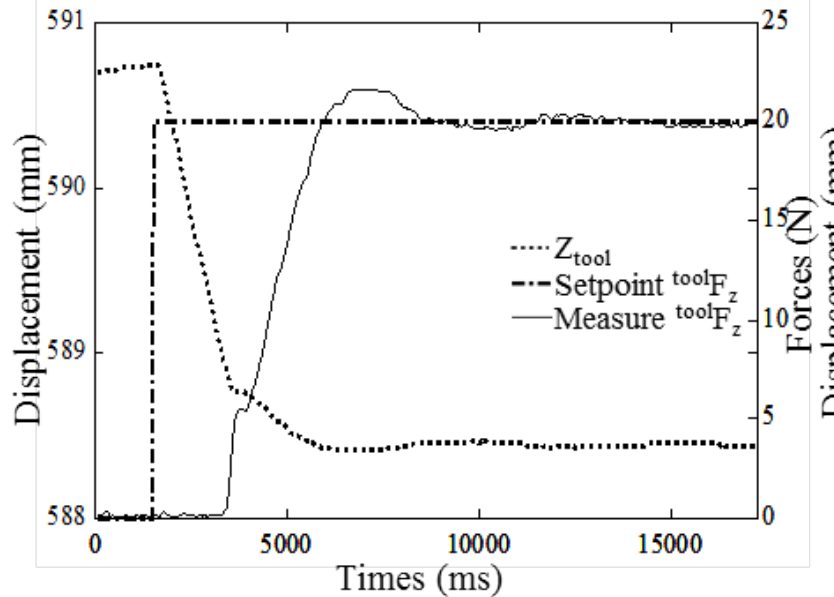

(a)

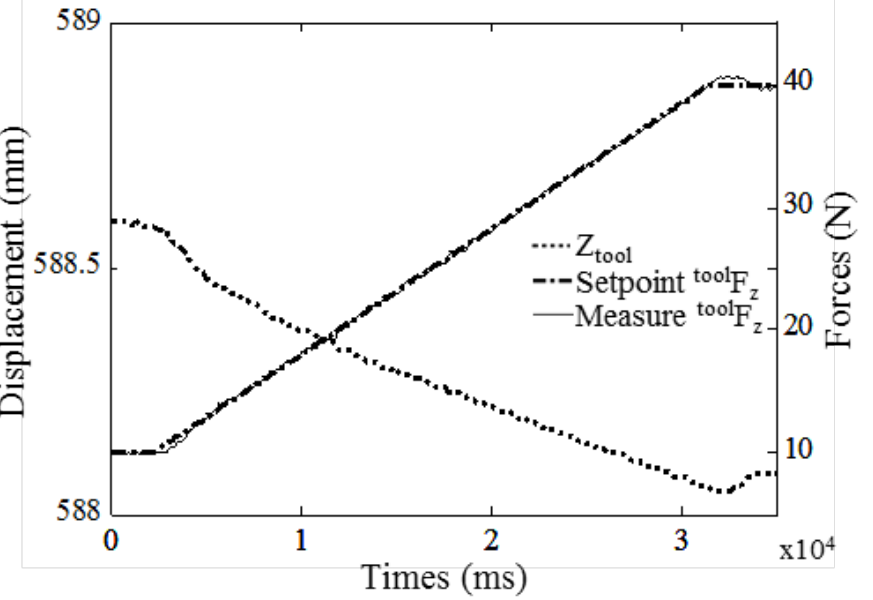

(b)

Fig. 18 Force servoing (a) with step input (b) with slope input

error is about $\pm 0.13 \mathrm{~N}$ and the maximum deviation is equal to $0.38 \mathrm{~N}(1.42 \%)$.

The PID parameters (for FSG) have been tuned experimentally and the system behaviour is good enough to add laying-up simulation using the position servoing along the $x$-axis.

\section{Force servoing with motion}

To observe the effect of force servoing on AFP task, we have setup two test scenarios for each experiment. We only change the fact that forces/moments are monitored or controlled. We simulate a laying-up process on a horizontal plate thus the $z$-coordinate of the FPT should remain at constant value in the workshop frame. The motion is only among the $x$-axis of the FPT frame and the displacement is $100 \mathrm{~mm}$.
Firstly, the system is controlled in position as the actual implementation, Fig. 19. When the tool moves along $x$-axis, (Fig. 19.a), the tool position along $z$-axis is almost constant (noise from kinematic model computation) and the compacting strength increases very much from $20 \mathrm{~N}$ to $45 \mathrm{~N}$ (Fig. 19.b). In fact, this shows that the assumptions of the surface being horizontal and flat are not true or the FPT is not well oriented to have its $z$-axis pointing vertically toward the ground. Moreover, one can notice that a force along $y$-axis appears with a mean value of $5 \mathrm{~N}$. This force should not appear if the compaction roller movement was a pure rolling one. This $y$-axis force will tend to generate an unwanted displacement among this axis. The unwanted $y$-axis displacement is the one which will lead to non respect of the gap between tapes during the laying-up.

Secondly, the system is controlled with hybrid position/force control mode, Fig. 20. Here, the tool posi- 


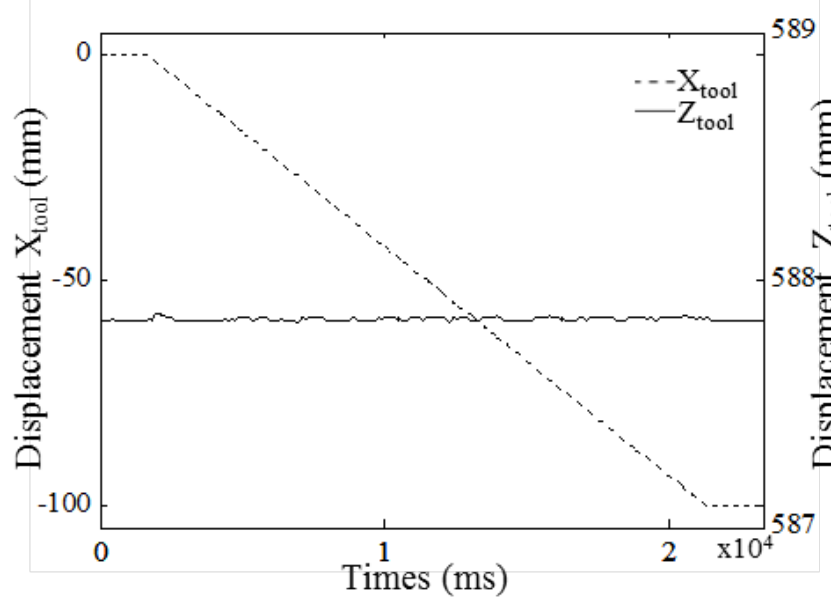

(a)

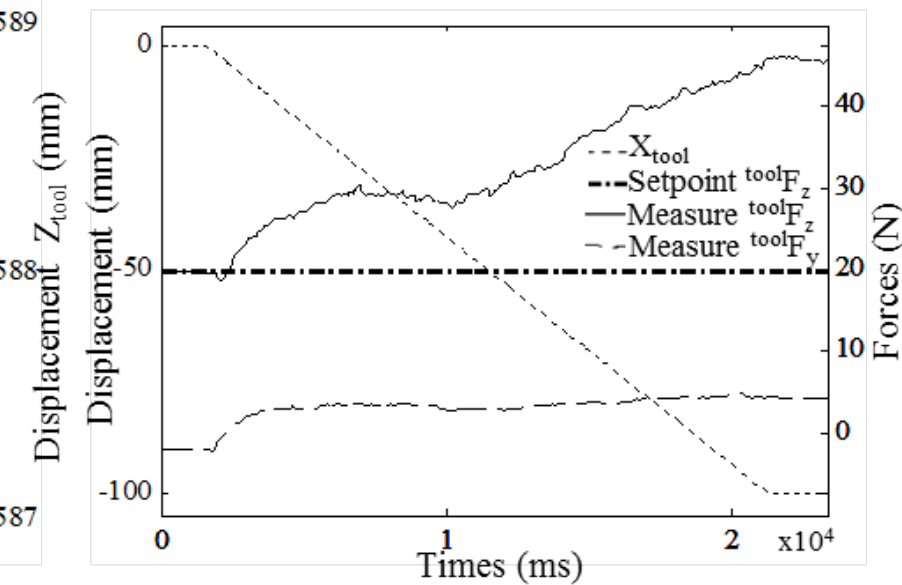

(b)

Fig. 19 Compacting strength with position control during motion

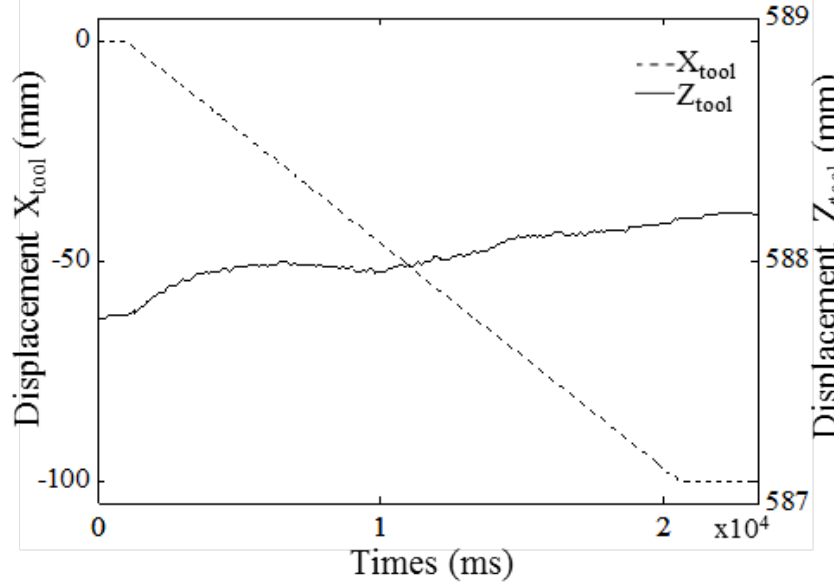

(a)

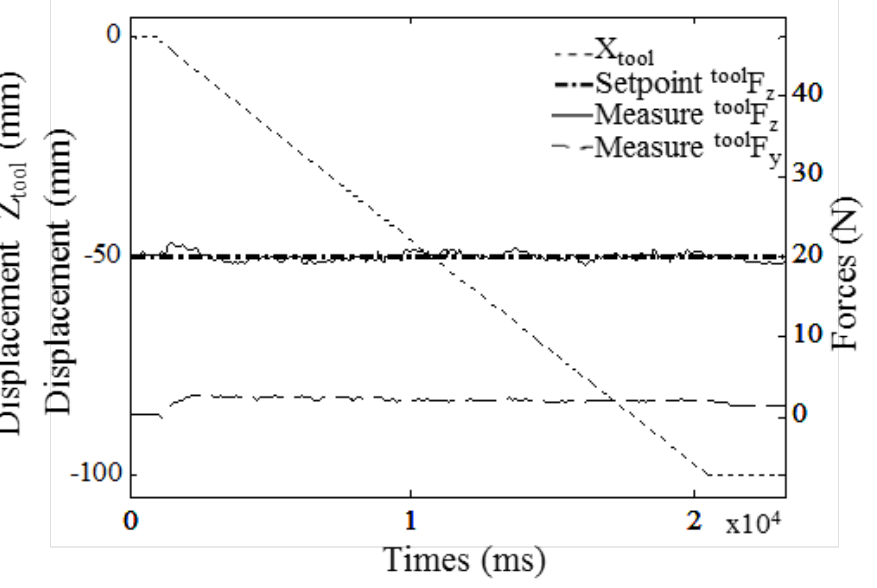

(b)

Fig. 20 Compacting strength with force control during motion

tion is corrected along $z$-axis of the FPT in order to maintain the compacting strength at the desired value thanks to the force servoing loop. The measured force along $z$-axis is kept around the $20 \mathrm{~N}$ target. In fact, the tool needs to move down around $\pm 0.25 \mathrm{~mm}$ along $z$-axis to keep the compacting strength and there are oscillations around the set-point of $20 \mathrm{~N}$ during the displacement. We can observe that the unwanted force along $y$-axis has decreased to $1.8 \mathrm{~N}$ (was $5 \mathrm{~N}$ without force control). With the force servoing, we have shown that the unwanted forces and moments on the position controlled directions are lower thus the trajectory of the fibre placement will be less perturbed.

\subsubsection{Position - Visual servoing}

Firstly, we present the image processing and the visual servoing control without other control neither in posi- tion or force. Secondly, we present the results obtained with the position and visual control.

\section{Edge detection and visual servoing}

As the vision system is an eye-in-hand one with an active lighting (laser beam), the image processing is a lot simpler than in a natural environment. Figure 21 shows the laser line projected on the carbon fibre. As the positioning of the triplet: laser source, camera and compacting roller is known the image processing can be done on a sub image and we do not need to run the processing on all image. In this sub image, the thickness change of $0.3 \mathrm{~mm}$ corresponds to 10 pixels. Thus, the processing time is kept as short as possible and a high image frame rate can be achieved. The image processing steps are the following:

- Colour image capture, 


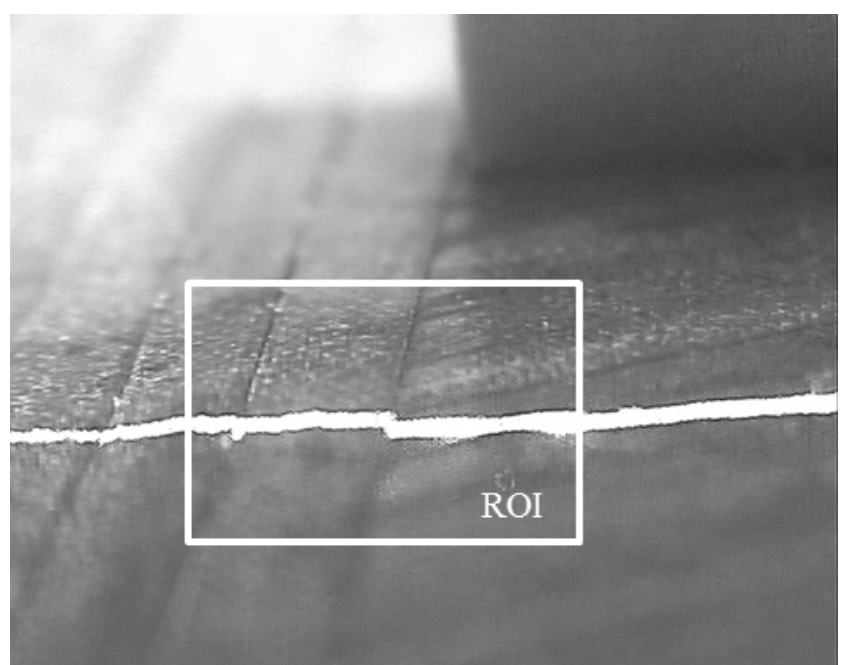

Fig. 21 Typical captured image

- Colour to Gray conversion,

- Binarization,

- Contour finding (Canny Edge filtering),

- Contour analysis (polyline approximation),

- Edge detection (thickness change in laser line).

Typical image processing result is shown in Fig. 22. One can see the polyline approximation of the contours find with the Canny Edge detector. The edge detection is located between the extremum.

We present the result obtained for the robot control among the $y$-axis while the other directions are locked. Thus, the VSG parameters (PID ones) can be tuned. The test is to set the target to a desired position in the image and then activate the visual servoing. On Fig. 23, we can see the target value set at 300 pixels, the edge detection and the $y$-axis displacement. The activation time is $1100 \mathrm{~ms}$. When the visual servoing is activated, the detected edge converges to the target value (precision is \pm 2 pixels).

The PID parameters (for VSG) have been tuned experimentally and the system behaviour is adequate to add laying-up simulation using the position servoing along the $x$-axis.

\section{Visual servoing with motion}

To be able to measure the improvement on tape-to-tape placement, we have setup two test scenarios for each experiment. We only change the fact that visual data are monitored or controlled. We simulate a laying-up process on a horizontal plate thus the $z$-coordinate of the FPT should remain at constant value in the workshop frame. The motion is only among the $x$-axis of the FPT frame and the displacement is $120 \mathrm{~mm}$.

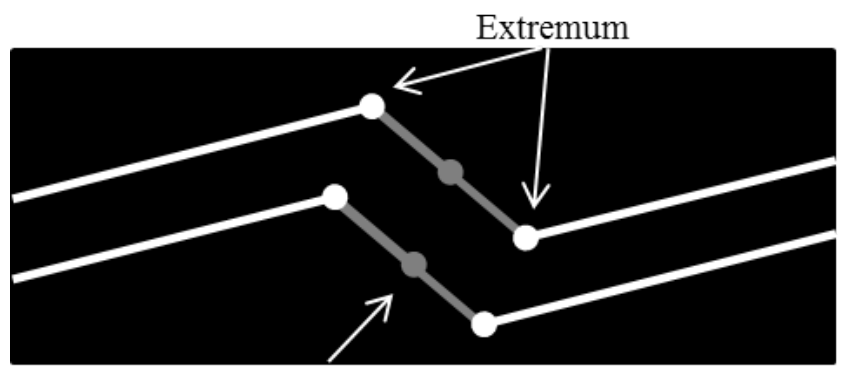

Edge detection

Fig. 22 Image processing results

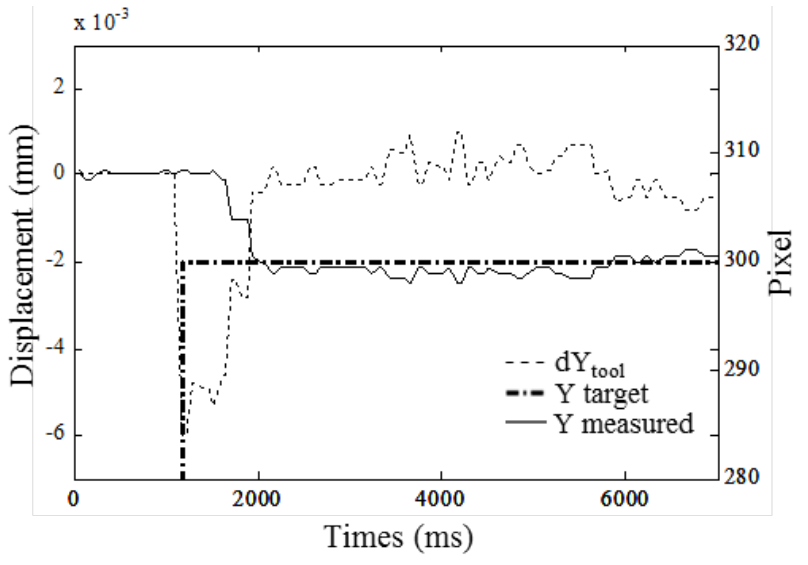

Fig. 23 Visual servoing step response

During this $x$-axis displacement, the position in pixel along $y$-axis of the detected edge is measured and compared to the position of the target. On Fig. 24, ones can notice the $x$-axis displacement $\left(X_{\text {tool }}\right)$ with a starting time sets at $2500 \mathrm{~ms}$, the edge detection during the motion ( $\mathrm{Y}$ measured) and the potential edge target ( $\mathrm{Y}$ target). Converting the pixel deviation into millimetres thanks to the camera calibration results, the tape gap shift is about $1.5 \mathrm{~mm}$ at the end of displacement.

In visual control mode, the lateral position of the compaction roller is controlled in closed loop. The robot is controlled in position following $x$-axis to make a displacement of 120 millimetres and it is controlled in visual servoing following $y$-axis to be aligned on the tape edge. The Fig. 25 represents the experimental results: the $x$-axis displacement $\left(X_{\text {tool }}\right)$ with a starting time sets at $2000 \mathrm{~ms}$, the edge detection (Y measured) during the motion and the edge target ( $\mathrm{Y}$ target). The visual servoing control scheme results are discussed for the tape-to-tape laying to demonstrate the improvements. When the system is controlled with hybrid position/visual control mode, the tool position is corrected along $y$-axis in order to maintain the detected edge on 
the image aligned to the target.

In average, the error mean value is equal to 2 pixels which represents $0.06 \mathrm{~mm}$. The maximum deviation is equal to \pm 12 pixels or $\pm 0.36 \mathrm{~mm}$ due to the edge detection. Moreover, we can observe that the displacement along $y$-axis with the hybrid position/visual control mode is a mirror of the position measured along $y$-axis in pixel on the image with the position control mode, Fig. 25.

The first results show that if the first tape position is not corresponding to the theoretical tape position, we obtain gap or/and overlap between the two tapes. However, the hybrid position/visual control has a positive impact on the system to improve its accuracy. In fact, the two tapes are aligned.

\subsubsection{Position - Force - Visual servoing}

As the fibre placement task need at the same time the compacting force, the geometrical trajectory of the tape within the ply and the inter-band gap control, we have implemented on our experimental setup the control scheme proposed in Fig. 11. In this test, the force servoing along $z$-axis and the visual servoing along $y$ axis are active during the motion of the tool along $x$ axis of $120 \mathrm{~mm}$. The system is controlled with hybrid position / force / visual mode. We simulate a laying-up process on a horizontal plate beside another tape.

In Fig. 26-a, we can see the force servoing of the compacting strength $\mathbf{F}_{z}$. The tool position is corrected along $z$-axis of the FPT in order to maintain the compacting strength at the desired value of $10 \mathrm{~N}$. The measured force along $z$-axis is kept around the $10 \mathrm{~N}$ target. The maximum deviation is equal to $\pm 2 \mathrm{~N}$. This value is almost constant with regards to the compacting strength (from $10 \mathrm{~N}$ to $40 \mathrm{~N}$ ). So the error decreases in percentage when the compacting strength setpoint increases.

In Fig. 26-b, we can see the visual servoing of the lateral position of the tape. The tool position is corrected along $y$-axis of the FPT in order to be aligned on the previous tape edge. The measured position of the tool in pixel on the image is kept around the 300 pixels target. The maximum deviation is equal to $\pm 0.3 \mathrm{~mm}$.

\section{Discussion}

We proposed the use of an external hybrid control scheme and a multi-sensors approach to improve the industrial process of fibre placement. After a process analysis, the
6 DOF of the task are defined as follow:

- 2 directions in force/moment to apply the compacting force (defined in task frame),

- 1 direction in vision to control the gap between tapes (according to the previous tape),

- 3 directions in position/orientation for the tape layingup (according to off-line programming).

To be able to run the task on a robot cell, we use two sensor based controls: generalized force control and visual servoing control. These control schemes have been tested separately, then integrated together and the results presented in the result section.

In the actual industrial system, there is no sensor feedback (neither force nor vision). The compacting strength is not guaranteed so the manufacturing procedure may not be respected. To avoid delamination between plies, the industrials tend to use larger values for the pneumatic cylinder control (open loop control). Due to interaction stiffness (compaction roller vs mould), a small difference (less than one millimetre) between the CAD model used for off-line programming and the real environment (despite a calibration), leads to force appearing in position controlled directions. These unwanted forces create displacements which make impossible a bidirectional laying-up (time wasting).

Compared to the actual industrial process, the improvements are mainly:

- a regulation of compacting force even if some perturbations occur,

- a better force distribution on the compaction roller,

- a diminution of unwanted external forces at the roller which reduces the displacement perturbations,

- a regulation of inter-band gap.

The proposed sensor based approach (force - vision) could make possible the bidirectional laying-up manufacturing (which reduces the manufacturing duration).

A weak point of our experimental setup is that the KUKA KRC2 controller only offers a 12 ms sampling period so laying-up speed is limited. But more modern industrial controllers like the KRC4 offer a $4 \mathrm{~ms}$ sampling period so the control can run three times faster. Another point to be improved is the edge detection to ameliorate the stability of the $y$-axis localisation of the previous tape. This could be addressed by a better suited image processing and also by using a better active vision device including a laser projector making a projected line with a width under the millimetre. 


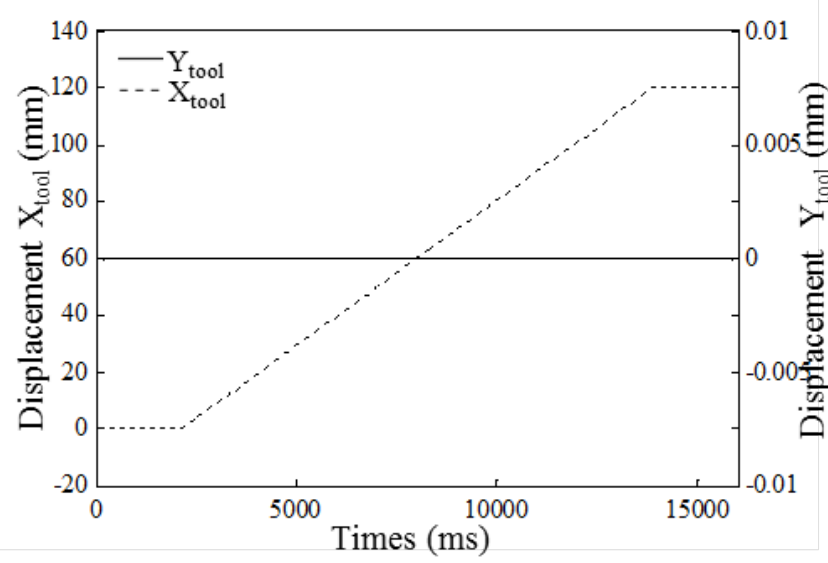

(a)

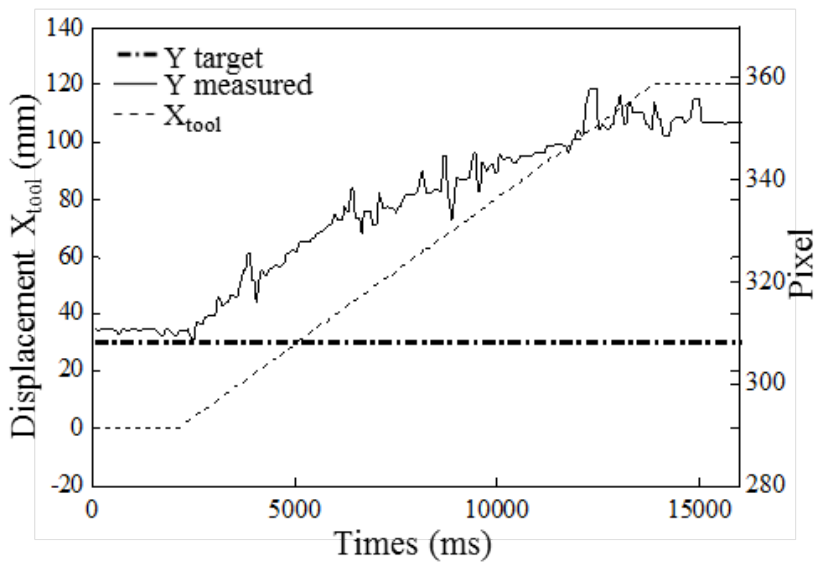

(b)

Fig. 24 Edge monitoring during $x$-axis motion

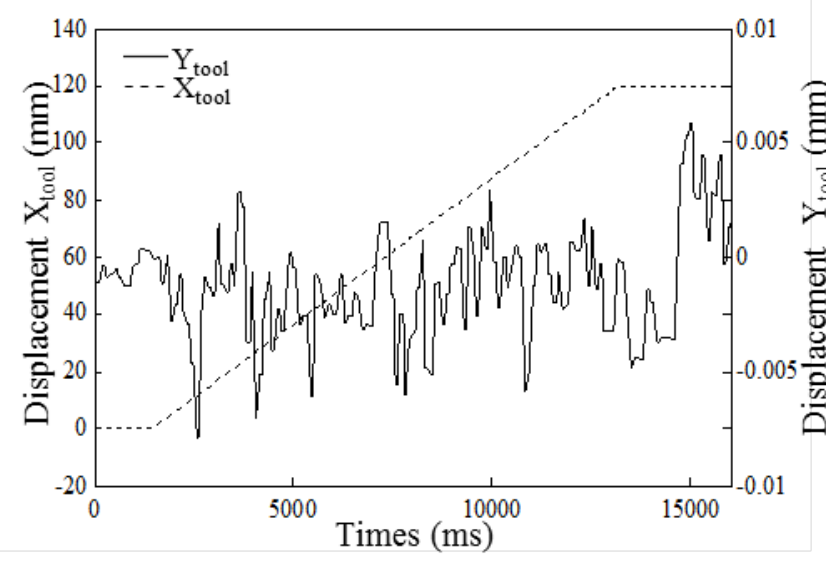

(a)

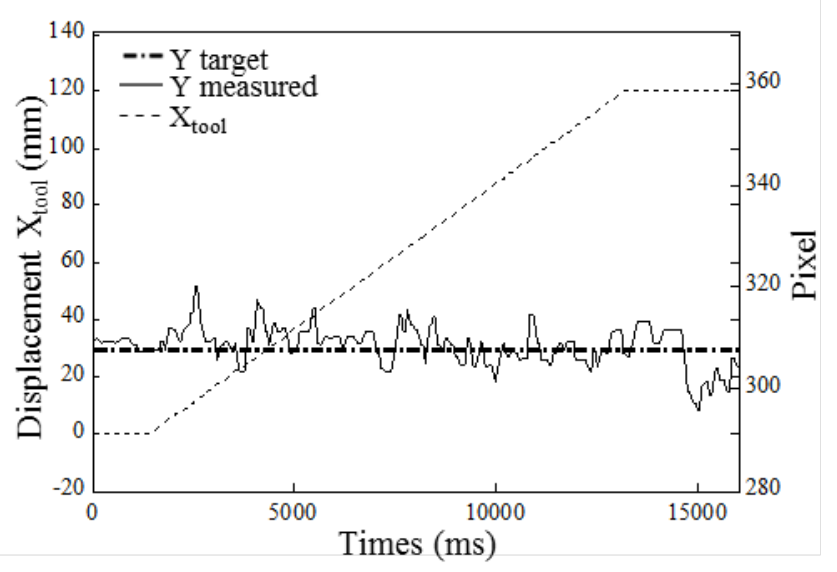

(b)

Fig. 25 Visual servoing along $y$-axis during $x$-axis motion

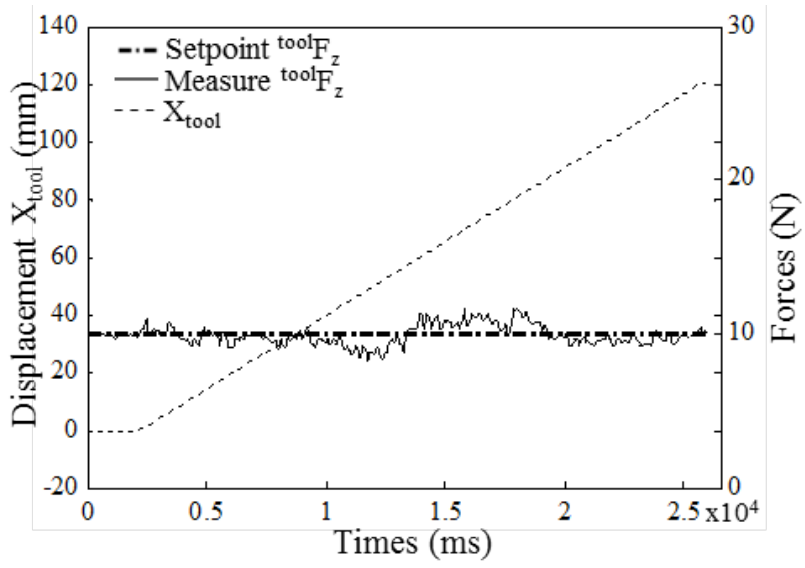

(a)

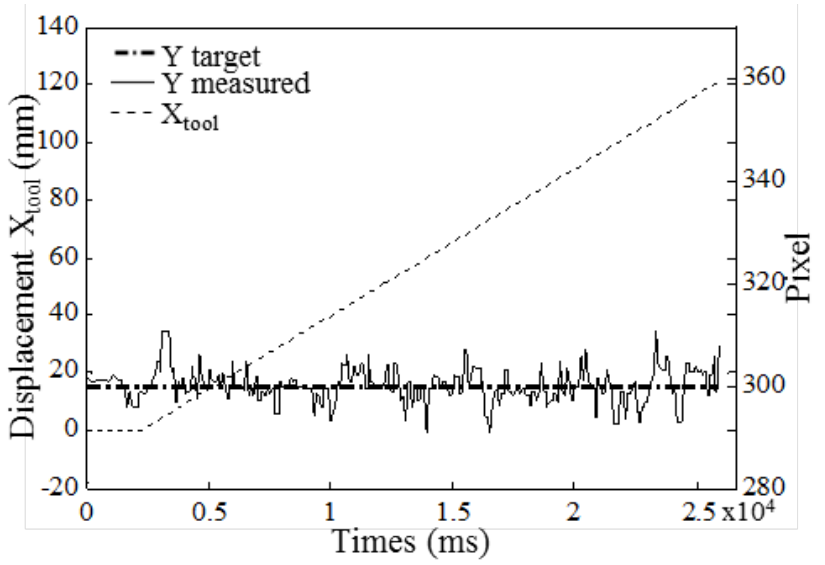

(b)

Fig. 26 Motion along $x$-axis with (a) force servoing along $z$-axis and (b) visual servoing along $y$-axis 


\section{Conclusion}

In the paper, we have presented a sensor based control scheme for an industrial process: Automated Fibre Placement. The industrial application is also giving us some technical constraints on the implementation. For example, the original robot controller can not be modified so some advanced control schemes cannot be used in our case. The placement fibre task is dual one: force and position servoing. By adding a force control scheme, the displacement disturbances are reduced compared to the standard control. Then, we proposed a visual control scheme to manage the lateral position of the tape in order to control the gap. Finally, we have three external control loops for position, force and visual servoing. The force control law and the visual control law have been tuned separately but an improvement can be achieved to take into account their interaction (one can be seen as a perturbation for the other). These exteroceptive loops can be seen as an interactive manufacturing scheme which permits to run off-line programming paths from CAM software.

Our future work will be more focused on transferring the work presented here to an industrial setup. The tests have been done on a laboratory robotics cell which is similar to the industrial one but scaled done in size and interaction forces. To do the knowledge transfer to industry, the control scheme need to be ported on the KR240 industrial robot with a force sensor capable of measuring the fibre placement compacting force up to 1000 N. It will be important to study how the change of range of physical parameters can influence the results of this paper and to see how to modify our control strategy to keep its efficiency.

The industrial cell is using a high power laser beam to heat the fibres to get the right sticking property, the laser light is composed of four length waves. To avoid perturbations at the camera level, we have planned to use a monochromatic optical filter, centred on the projection-laser beam frequency, to block the heating laser ones.

\section{List of Figures}

1 Robotic cell for Automated Fibre Placement . . . . . . . . . . . .

2 Laying-up process: a) Side view; b) Front view; c) Top view . . . . . . . . . . . . . 5

$4 \quad \mathrm{MDH}$ parameters .......... 5

3 Geometric architecture . . . . . . . . . 6

5 General control scheme........ 7

6 Position servoing scheme . . . . . . . 8

7 Task frame and transformation of moment 9

8 Force Servoing Scheme . . . . . . . . . 10

9 Thickness detection . . . . . . . . . . 10

10 Visual servoing scheme . . . . . . . . 10

11 Position-force-visual servoing scheme . . 12

12 Test environment . . . . . . . . . . . . . 13

13 GUI of the application . . . . . . . . . 14

14 View of the 3D simulator . . . . . . . 15

15 Active vision frames description . . . . . 16

17 Camera, laser beam setup on the FPT . 16

16 Active vision optimization . . . . . . . 17

18 Force servoing (a) with step input (b) with slope input . . . . . . . . 17

19 Compacting strength with position control during motion . . . . . . . . 18

20 Compacting strength with force control during motion . . . . . . . . . . 18

21 Typical captured image . . . . . . . . . . 19

22 Image processing results . . . . . . . . . . 19

23 Visual servoing step response . . . . . . 19

24 Edge monitoring during $x$-axis motion . 21

25 Visual servoing along $y$-axis during $x$ axis motion . . . . . . . . . . . 21

26 Motion along $x$-axis with (a) force servoing along $z$-axis and (b) visual servoing along $y$-axis . . . . . . . . . . . 21

\section{List of Tables}

1 Modified Denavit-Hartenberg Parameters [17] for Fiber Placement Robot . . . . .

2 Selection matrices following the steps of lay-up ...............
3

5

(5)

7

(1)

(1)

2

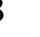

4

6

7

7

18

8
9
9
9
1




\section{References}

1. Baeten, J., De Schutter, J.: Improving force controlled planar contour following using online eye-in-hand vision based feedforward. In: Proc. IEEE/ASME Int. Conf. on Advanced Intelligent Mechatronics (Cat. No.99TH8399), pp. 902-907. Atlanta, Georgia (1999)

2. Bouguet, J., P. Perona: Camera calibration from points and lines in dual-space geometry. In: Proc. of the 5th European Conference on Computer Vision. Freiburg, Germany (1998)

3. Breteau, T., Damay, T., Duc, E., Hascoët, J.Y.: Design for manufacturing with tool paths adapted to marine propeller. International Journal on Interactive Design and Manufacturing (IJIDeM) 5(4), 271-275 (2011)

4. Bruyninckx, H., De Schutter, J.: Specification of forcecontrolled actions in the "task frame formalism". A synthesis. IEEE Trans. on Robotics and Automation 12(4), 581-589 (1996)

5. Carelli, R., Oliva, E., Soria, C., Nasisi, O.: Combined force and visual control of an industrial robot. Robotica 22, 163-171 (2004)

6. Chalaye, H.: Les matériaux composites : Dynamisme et innovation. SESSI, DiGITIP, Les 4 pages des statistiques industrielles, n158 (2002)

7. Corke, P.: Visual control of robots: High performance visual servoing. Research Studies Press LTD, Australia (1996)

8. Dombre, E., Duchemin, G., Poignet, P., Pierrot, F.: Dermarob: a safe robot for reconstructive surgery. IEEE Trans. on Robotics and Automation 19(5), 876-884 (2003)

9. Drake, S.H.: Using compliance in lieu of sensory feedback for automatic assembly. Ph.D. thesis, Massachusetts Institute of Technology (1978)

10. Espiau, B., Chaumette, F., Rives, P.: A new approach to visual servoing in robotics. In: Proc. IEEE on Robotics and Automation, vol. 8, pp. 313-326. IEEE (1992)

11. Fantini, M., De Crescenzio, F., Ciocca, L.: Design and manufacturing of customized surgical devices for mandibular rehabilitation. International Journal on Interactive Design and Manufacturing (IJIDeM) 7(4), 227$237(2012)$

12. Hachemane, B., Bezzazi, B.: Material composite fatigue behavior. In: Proc. 16ème Journées Nationales sur les Composites. Toulouse, France (2009)

13. Hebert, P., Hudson, N., Ma, J., Burdick, J.: Fusion of stereo vision, force-torque, and joint sensors for estimation of in-hand object location. In: Proc. IEEE Int. Conf. on Robotics and Automation, pp. 5935-5941. Shanghai, China (2011)

14. Hogan, N.: Stable execution of contact tasks using impedance control. In: Proc. IEEE Int. Conf. on Robotics and Automation, vol. 4, pp. 1047-1054. Raleigh, NC, USA (1987)

15. Hosoda, K., Igarashi, K., Asada, M.: Adaptive hybrid control for visual and force servoing in an unknown environment. IEEE Robotics \& Automation Magazine 5(4), 39-43 (1998)

16. Khalil, W.: Asservissement visuel. In: La commande des robots manipulateurs, hermes edn., chap. 3, pp. 105-150. Paris, France (2002)

17. Khalil, W., Dombre E.: Modélisation, identification et commande des robots. Hermes Sciences, Paris, France (1999)

18. Kulpate, C., Mehrandezh, M., Paranjape, R.: An eyeto-hand visual servoing structure for $3 \mathrm{D}$ positioning of a robotic arm using one camera and a flat mirror. In: Proc. IEEE/RSJ Int. Conf. on Intelligent Robots and Systems, pp. 1464-1470. Alberta, Canada (2005)

19. Lipiello, V., Siciliano, B., Villani, L.: Robot Force/Position Control with Force and Visual Feedback. In: Proc. European Control Conference, pp. 3790-3795. Kos, Greece (2007)

20. Lucas, T.: La dynamique des nouveaux procédés. L'Usine Nouvelle, n3093 (2008)

21. Luo, R.: Multisensor fusion and integration: approaches, applications, and future research directions. IEEE Sensors Journal 2(2), 107-119 (2002)

22. N. J. Johnston, H. L. Belvin, R. J. Cano, J. M. Marchello, A. B. Hulcher: A prototype research laboratory for automated fabrication of high performance composites. In: Proc. of the 12th International Conference on Composite Materials. Paris, France (1999)

23. Nadeau, J.P., Fischer, X.: Research in Interactive Design (Vol. 3): Virtual, Interactive and Integrated Product Design and Manufacturing for Industrial Innovation. Springer Science \& Business Media (2011)

24. Nagata, F., Hase, T., Haga, Z., Omoto, M., Watanabe, K.: CAD/CAM-based position/force controller for a mold polishing robot. Mechatronics 17(4-5), 207-216 (2007)

25. Ouadah, N., Hamerlain, M., Djouadi, M.S.: Positionnement d'un bras manipulateur par une commande référencée vision. In: Conf. Int. sur les systèmes de Télécommunications d'Electronique Médicale et d'Automatique. Tlemcen, Algérie (2003)

26. Pages, J., Collewet, C., Chaumette, F., Salvi, J.: Optimizing plane-to-plane positioning tasks by image-based visual servoing and structured light. IEEE Trans. on Robotics and Automation 22(5), 1000-1010 (2006)

27. Prats, M., Sanz, P.J., Pobil, A.P.: Reliable non-prehensile door opening through the combination of vision, tactile and force feedback. Autonomous Robots 29(2), 201-218 (2010)

28. Raibert, M.H., Craig, J.J.: Hybrid Position/Force Control of Manipulators. J. of Dynamic Systems, Measurement, and Control 103(2), 126 (1981)

29. Robertsson, A., Olsson, T., Johansson, R., Blomdell, A., Nilsson, K., Haage, M., Lauwers, B., Baerdemaeker, H., Brogardh, T., Brantmark, H.: Implementation of Industrial Robot Force Control Case Study: High Power Stub Grinding and Deburring. In: Proc. IEEE/RSJ Int. Conf. on Intelligent Robots and Systems, pp. 2743-2748. Beijing, China (2006)

30. Salisbury, J.: Active stiffness control of a manipulator in Cartesian coordinates. In: Proc. of the 19th IEEE Conf. on Decision and Control including the Symposium on Adaptive Processes, pp. 95-100. Stanford, California (1980)

31. Smith, C.E., Brandt, S.A., Papanikolopoulos, N.P.: EyeIn-Hand Robotic Tasks In Uncalibrated Environments. IEEE Trans. on Robotics and Automation 13, No.6, 903-914 (1997)

32. Smits, R., Bruyninckx, H., Meeussen, W., Baeten, J., Slaets, P., Schutter, J.: Model Based Position-ForceVision Sensor Fusion for Robot Compliant Motion Control. In: Proc. IEEE Int. Conf. on Multisensor Fusion and Integration for Intelligent Systems, pp. 501-506. Heidelberg, Germany (2006)

33. Talaba, D., Antonya, C., Stavar, A., Georgescu, V.: Virtual reality in product design and robotics. In: Proc. of International Conference on Cognitive Infocommunications, pp. 1-6. Budapest (2011) 
34. Uhart, M., Patrouix, O., Aoustin, Y.: Controlling the tape's gap in robotized fiber placement process using a visual servoing external hybrid control scheme. In: Proc. of the International symposium Aircraft Materials ACMA2014. Marrakech, Morocco (2014)

35. Uhart, M., Patrouix, O., Aoustin, Y., Canou, J.: Improving Accuracy in Robotized Fiber Placement. In: Proc. of The 19th International Conference on Composite Materials. Montreal, Canada (2013)

36. Whitney, D.: Historical perspective and state of the art in robot force control. In: Proc. IEEE Int. Conf. on Robotics and Automation, vol. 2, pp. 262-268. Cambridge, United States (1985)

37. Witten, E., Jahn, B., Karl, D.: Composites Market Report 2012 : Market developments, trends, challenges and opportunities. Tech. rep., AVK - Industrievereinigung Verstärkte Kunststoffe (Federation of Reinforced Plastics), Frankfurt, Germany (2012)

38. Xu, J., Xi, N., Zhang, C., Shi, Q.: Real-time 3D shape measurement system based on single structure light pattern. In: Proc. IEEE Int. Conf. on Robotics and Automation, pp. 121-126. Anchorage, Alaska (2010)

39. Zemiti, N.: Commande en Effort des Systèmes Robotiques pour la Chirurgie Mini-Invasive. Ph.D. thesis, Pierre et Marie CURIE - UPMC, Paris, France (2005)

40. Zheng, Y., Fan, Y.: Robot force sensor interacting with environments. IEEE Trans. on Robotics and Automation 7(1), 156-164 (1991)

\section{Acknowledgments}

This research work was done in the frame of the IMPALA project, a French funded project from FUI-011 program, under the convention number F 1108008 B. The project consortium is composed by Coriolis Composites, Dassault Aviation, Daher-Socata, Composites Aquitaine (an EADS Sogerma company), CompositAdour, Ecole Centrale de Nantes and ESTIA. 